\title{
PII S0271-5198(96)00081-X
}

\author{
C L I N I C A L $\quad$ H E M O R H E O L O G Y
}

CUMULATIVE AUTHOR INDEX AND SUBJECT INDEX

VOLUMES
$1989-1996$

Compiled by Inge Fisher

Executive Editorial Office, CLINICAL HEMORHEOLOGY

Diakonissen-Krankenhaus, Diakonissenstr. 28,

D-76199 Karlsruhe, Germany 


A U T H
$\mathrm{O} \quad \mathrm{R}$
I N
D E X

Aarnoudse J.G. 9, 553

Aarts $\underline{9}, 593$

Aarts P.A.M.M. $\underline{9}, 463,583$

Abdallah K. $\underline{9}, 371,11,227$

Abugo O. 15, 877

Acciavatti A. $\underline{9}, 459, \underline{10}, 171, \underline{11}, 361, \underline{13}, 177,687$, $14,709,15,221,367$

Achhammer G. 10,157

Acikgoz B. 13, 459

Adams R.A. 14, 149

Adewuyi J.O. 13,737

Adjizian J.L. $\underline{9}, 162,165$

Aellig A. 11, 465

Affeld K. 14, 421

Agashe V.S. 9 , 199,373,14, 321

Agosti, R. $\underline{9}, 89,439,823,16,559$

Agostoni A. $9,633,11,447$

Aguiar-Andrade A. $\underline{9}, 652$

Aichner F. 14, 11

Ajmani R. 12, 579

Ajmani R.S. 15,347

Akbas A. 13, 49

Akedo Y. 11, 351

Akiyama M. $\underline{9}, 371, \underline{12}, 935$

Al-Dakak I. 무 371, 11, 227

Al-Khaja N. 12, 229

Al-Min A. 11, 227

Al-Zahrani $\bar{H}$. 12, 867

Alamin A. $\underline{9}, 371$

Allegra C. $\underline{9}, 369,439, \underline{14}, 413$

Alletto G. 13,83

Allix M. $\underline{9}, 339$

Aloeva M. $\underline{15}, 783$

Altman D.G. 9,181

Altmann Ch. 11,303

Aman J. 9, 603

Amara F. $\underline{9}, 371, \underline{11}, 227$

Ambrus C.M. $\underline{9}, 440$

Ambrus J.L. $\underline{9}, 440, \underline{10}, 225$

Ameriso S.F. $\underline{9}, 441$
Amiral J. 14, S1

Amodio L. 15, 73

Amoussou Guenou K. 15, 167

Anadere I. $\underline{9}, 569,831, \underline{10}, 363, \underline{12}, 243$

Anain J.M. 10, 225

Anain S.M. 10, 225

Andac S.O. 오 $577, \underline{10}, 255,485, \underline{11}, 19$

Anderson J. $\underline{9}, 646, \underline{12}, 757$

Anderson K.W. 13, 671

Andreu G. 12, 455

Angelkort B. $\underline{9}, 441$

Annapurna V. $\underline{9}, 199, \underline{10}, 95,329, \underline{11}, 243$

Antonini V. 9,369

Antonova N. 15,45

Anwar M.A. $\underline{9}, 442, \underline{14}, 93,697$

Aoshiba K. 13, 23

Aouane F. 16, 677

Ardenne M. von $\underline{9}, 442$

Arend O. 11, 623, 12, 437,725, 13, 435, 16, 357

Arfors K.-E. 11, 417

Arnatz H.-R. 10, 297

Arnim von T. 11,575

Arntz H.R. $\underline{9}, 443, \underline{11}, 63$

Aronson H.B. 10,65

Artmann G.M. $\underline{9}, 443,444 \underline{13}, 529, \underline{15}, 133$

Aspelin P. 12, 401

Atakan N. $\underline{13}, 49$

Atger V. 15,667

Athanassiou G. $\underline{13}, 767$

Attali J.R. 15, 325

Aull S. 14, 339

Aurisch R. 9 , 444

Avellone G. 13,83

Aznar J. 11, 133,13, 447,14, 419,15, 677

$$
\underline{16}, 235,277,515,549
$$

Bach R. 11, 657

Bachelard B. 13, 215

Badiali M. 11,641 
Bädorf D. 13,501

Bagge U. 11, 369

Bahut C. 12, 357

Bailey R.R. $\underline{9}, 370$

Baird A.J. $\underline{9}, 973$

Baisheng W. 11, 237

Baisheng Wang 16, 603

Baker C.W. 13, 671

Balzereit A. $\underline{9}, 329$

Barbas J.M. $\underline{9}, 648,649$

Barberá J.L. 16, 515

Barbounaki St. 15, 107

Bardet L. $\underline{13}, 651, \underline{15}, 191$

Barista I. 16, 313

Barnes A.J. 13, 661, 16, 479

Barnikol W.K.R. $\underline{9}, 445$

Barré J. 13,779

Barros F. $\underline{9}, 652$

Barshtein G. 14, 497

Bartholomeus I. 12, 217

Bartosz G. 10, 561

Baskova L.P. 15, 633

Baskurt O.K. 9, 577, 10, 255,485, 11, 19,121,497 $\underline{12}, 689, \underline{13}, 49,459, \underline{14}, 591$ $\frac{12}{15}, 97,16,49$

Battistelli S. $13,13,16,57,245,653$

Batuman V. 11, 295

Bauersachs R. 12, 841

Bauersachs R.B. $\underline{9}, 1,55$

Bauersachs, R.M. $\underline{9}, 446,723,935$

Baumgartner Ch. 10,499

Bäumler H. $\underline{9}, 445,460, \underline{10}, 645$

Baurain R. 14, $\mathrm{S} 15$

Beamer N.B. $9,273,11,167$

Beaty M.M. 13,671

Beauplet A. $\underline{9}, 460$

Beilin B. 10,65

Beillot J. 9, 625

Belboul A. 12, 229

Belch J.J.F. 10, 263

Belhoul A. 15, 641

Bellingham A.J. 10, 35

Bellù R. 12, 573

Benarroch I.S. $\underline{9}, 376$

Benezis C. 15,201

Benichou A.C. 15, 167

Bennett E.D. 10, 287

Berbenel J.C. 9,646

Berends G. 16,151

Berg R. 16, 343

Berger T. 16, 271

Berglöff J. 14, 45

Bergmann H. 10,525

Beritashvili N. 15, 697

Berling C. $\underline{9}, 446,447$
Bernasconi C. $\underline{9}, 633, \underline{11}, 447$

Berne C. $\underline{9}, 603$

Berni S. $\underline{9}, 656,11,41$

Berthault M.-F. 14, 83

Bertilli P. 12, 697

Bertolini S. 11, 9

Bertram B. $11,623, \underline{12}, 437, \underline{16}, 357$

Bertrand A. $\underline{9}, 163,164,237,855,14,19$

Beuzard Y. $\underline{9}, 451, \underline{11}, 55, \underline{12}, 415$

Beylot C. $\underline{9}, 871, \underline{15}, 627$

Beylot J. 15, 627

Bezencon M.-L. 10, 215

Bhatt J.A. 12, 427

Bicchi M. 12, 697

Billerey M. $\underline{9}, 163,10,321$

Billett H.H. 10, 35

Bilo H.J.G. $\underline{9}, 1017,13,465$

Bilto Y.Y. $\underline{9}, 447, \underline{15}, 797$

Binsack R. 15, 763

Birk A. 14,355

Birk A. 15, 681

Bitbol M. $\underline{9}, 281$

Black R.A. 12,45

Blardi P. $\underline{9}, 459, \underline{10}, 171, \underline{13}, 177$

Blom H.J. 9, 448

Blombäck B. 16, 383

Bodansky J. 9,454

Bode V. 15,359

Boemi M. $\underline{9}, 450,653,654, \underline{11}, 641, \underline{15}, 73, \underline{16}, 543$

Boeva D. 15,45

Böhler Th. $\underline{9}, 449, \underline{13}, 67,775, \underline{15}, 305$

Boisseau M. $\underline{9}, 861$

Boisseau M.R. 9, 161, 162, 181, 450, 451, 861,

$871,11,655, \underline{14}, 19,171, \mathrm{~S} 1, \underline{15}, 627, \underline{16}, 27$

Boivin P. 14, 819

Boldt J. 12, 71

Bollati P. $\underline{9}, 655$

Bompiani G.D. 13,83

Bondoux G. $9,163,237,543,845,861$

Bonifacio M. $\underline{9}, 369,439$

Bonillo A. 14, 75

Bonitchi P. 11, 649

Bonne C. 9, 209, 12, 287

Bonnier J.J.R.M. 12, 217

Borgogni T. 16, 57, 245, 653

Boroda C. $\underline{9}, 372,11,191$

Borovetz H.S. 11, 325

Bosquet F. 9, 281

Botas L. 9, 651, 656

Botzenhardt R. 11, 575

Bougaret S. 14, S7

Boulot P. 13, 215, 14, 637, 643, 15, 13

Boulu R.G. 9, 339

Bourbigot B. 13, 261

Bournier O. 14, 819 
Bouskela E. 14, S23

Boynard M. $\underline{9}, 159,451,771,11,55,14,117,255$

Bozzoni M. $\underline{9}, 89$

Bracci R. $\underline{9}, 656,11,41$

Bradiali M. $\underline{9}, 653$

Braide M. 11, 369

Braun M. $\underline{9}, 328$

Braun M.E. $\underline{9}, 452$

Bravo M.L. 11, 339

Bregni C. $\underline{9}, 559$

Breugel H.H. $\underline{9}, 453$

Breugel H.H.F.I. 9,452

Brockmann M. 12, 237

Bromberger U. $\underline{9}, 462$

Bronkhorst P.J.H. 15, 803, 16, 151

Brooks D.E. $\underline{9}, 695$

Brossay L. 9,164

Brown J.R. 11, 441

Brown M. J. 10, 491

Brown P. $\underline{9}, 139$

Brückner U.B. 10,383

Brugnara C. 12,177

Bruhn H.D. $\underline{9}, 329$

Brun J.F. $\underline{9}, 166,351,361,953,13,187,201$, $215,651,14,105,637,643,807$

Bruneau C. 11, 583 $15,13,147,191,201,213$

Bryce F.C. 9,454

Brzezinska Z. 15,641

Buccianti N. 13, 687

Buchan P.C. $\underline{9}, 453,454, \underline{14}, 651$

Bucherer C. $\underline{9}, 160,11,387,12,415,511,733$

Buffet E. $\underline{9}, 162$

Bugl R.B. $\underline{9}, 454$

Bull B.S. 12,407

Buonocore G. $\underline{9}, 656, \underline{11}, 41$

Buonocore M. $\underline{9}, 163$

Burroni L. 12, 697

Buscemi S. 9,456

Busquet M. $\underline{9}, 161,451,871$

Butsch S. $\underline{9}, 440$

Caglayan S.S 10, 255,485, 11, 121,497

Cai Shaoxi 16, 135

Caillot A. 15, 167

Caimi G. $\underline{9}, 373,455,456,839,10,449,11,1,41$, $617,12,449,13,35,227,14,393,597$ $15,53,613,657, \underline{16}, 177,523,685$

Calabrò A. 11, 405

Camberlein V. 11, 583

Campion J.C. $\underline{9}, 166,458$

Candiloros H. 12, 873

Canino B. $9,455,11,1,14,393,597$, $\underline{15}, 53,657, \underline{16}, 177,523$
Cantera L. 13,237

Cao Shi 11, 459,507

Capecchi P.L. 13, 687, 14, 709, 15, 221

Cappelli R. 12, 697, 13, 13

Cardose L. $\underline{9}, 647,648,649,650,651,656$

Carlizza A. $\underline{9}, 369,439, \underline{14}, 413$

Carmena R. 13, 447

Carnevale Schianca G.P. $\underline{9}, 654$

Carter C. 12, 109

Cartry E. ㅇ, 351

Carvalho Sousa J. 9,652

Castellini M.A. 12, 657

Castenholz A. 16, 577

Caswell M. 10, 275, 12, 309,407

Catania A. $\underline{9}, 373,455,456, \underline{10}, 185,449,13,35$ $227, \underline{14}, 393, \underline{15}, 53,657, \underline{16}, 177,523$

Ceccatelli L. $13,687,15,221$.

Cerasola G. 13,35

Chabanel A. $\underline{9}, 456, \underline{12}, 455, \underline{14}, 401, \underline{15}, 381$

Chalder S.M. $\underline{9}, 1009$

Chandalia H.B. $\underline{9}, 373,11,243$

Chantachum Y. 15, 729

Chappey O. 16, 661

Charpiat A. 15,147

Chen H.-Q. 11,635

Chen Hao 16, 135

Chen Huai-Quing 11, 25

Chen Ji-di 15, 81

Chen Li $\underline{9}, 615$

Chen S. 14,497

Chen S.C. 15,775

Chen T.Z. 15, 775

Cherubini P. $\underline{9}, 89$

Chien S. 10, 191

Chirkova L.D. 15,633

Chisholm A.B.J. $\underline{9}, 377$

Chmiel H. 9 , $327,569,10,363$

Cholerton S. 11, 441

Christakis J. 10,35

Christopher B.D. 10,113

Chu G. 9,165

Chung T. 10, 505

Chung T.W. 15, 775

Chungling D. 11, 237

Chuttner J. 10, 191

Cietto S. 12,573

Ciszewski R. 10, 561

Clerc J.M. $\underline{9}, 164$

Clerck de P. $\underline{9}, 645$

Clivati A. $\underline{9}, 89,439,823$

Closse C. 14, $\mathrm{S1}$

Coccheri S. 10, 321

Coce F. $\underline{9}, 965$

Colin F.C. 13,261

Colombo T. 12, 79 
Comes K. $\underline{9}, 458$

Conan F. $\underline{9}, 47$

Conri C. $14,51,15,627$

Constans J. 14, S1, 15, 627

Contorno A. 13,35

Cooke B.M. 15,377

Copley A.L. $\underline{9}, 395,809,10,3, \underline{11}, 149,271$

Corda C. 14,663

Cordova R. 13,83

Corlett M.P. 12, 407

Cortese C. 9,81

Coull B.M. $\underline{9}, 273,374, \underline{11}, 167$

Counord J.-L. 14, 83

Craveri A. $\underline{9}, 655, \underline{10}, 541, \underline{12}, 573$

Cravino J. $\underline{9}, 650$

Cravo M. 9,647

Cremel G. 11, 583

Crepaldi G. 11, 405

Criqui C. 9,351

Criscuolo A. 9 , 369, 439

Cristal N. $\underline{9}, 650,651$

Crnek S. 10,477

Crombie I.K. $\underline{9}, 646$

Crouch S.P.M. 14,379

Cruz J. $\underline{9}, 650$

Cuiffetti G. $\underline{9}, 457$

Culiolo B. $\underline{9}, 165$

Culliford S.J. 14, 545

Cunha J.C. 9,650

Cunha Ribeiro LM. $\underline{9}, 652$

Cuschieri R. 12,867

Cyrino F.Z.G.A. 14, S23

D'Alfonso A. 9,999

D'Asaro S. $\underline{9}, 455,456, \underline{10}, 449, \underline{11}, 1,49, \underline{13}, 227$

D'Ettorre M. $\underline{9}, 823$

Daburger L. 11,429

Daga S.R. $\underline{9}, 199$

Dakwa C. 13,737

Dal Frà M.G. 11, 405

Dalmau J. 16,549

Damiani P. $\underline{9}, 459,10,171,14,709$

Danon D. $\underline{9}, 651$

David M. 12, 67

Davidson R.J.L. $\underline{9}, 115, \underline{10}, 21,51$

Dawson P. 12,369

De Ceulaer K. 10, 35

De Franco V. 10, 171

De Giorgi L. 13, 687, 14, 709

De Lalla A. 15,221

De Leeuw I. $\underline{9}, 983$

De Matteis C. 14,797

De Matteis F. 14, 203

De Matteis G. $\underline{9}, 999$
De Simone R. 13,83

Deecke L. 10, 499

Deeke L. $\underline{16}, 285$

Dehn R. 16, 249

Del Santo A. 11, 447

Del Santo A. $\underline{9}, 633$

Delamaire M. $\underline{9}, 458,460$

Delomier Y. $\underline{15}, 167$

Delsignore R. 11,605

Demiroglu C. 16, 271,

Demiroglu H. $\underline{16}, 313$

Dempsey R.J. 13, 671

Deng, Ch. $\underline{9}, 369$

Denison D. 13,473

Deo K. 12, 427

Derham R. $\underline{9}, 453$

Derriennic X. 12, 873

Desai A.P. 14, 223

Deschamps F. 13,215

Devi B.L. 12, 767

Dezier J.F. $\underline{9}, 625$

Dhar P. 15,715

Dhermy D. 14,819

Di Bella M. $\underline{9}, 655$

Di Garbo V. 13, 83

Di Massimo C. 14, 203

Di Massimo C. $\underline{9}, 999$

Di Perri T. $\underline{9}, 459, \underline{10}, 171, \underline{11}, 361, \underline{13}, 177,687$, $14,709, \underline{15}, 221,367$

Diebold U. $\underline{9}, 329$

Dikmenoglu N. 10, 255, 11, 121, 497, 13, 49

Dintenfass L. $\underline{9}, 367,374,677, \underline{10}, 411, \underline{11}, 155$

Distler B. $\underline{9}, 445,460, \underline{10}, 645$

Dittmann K. $\underline{9}, 444$

Dobbe J.G.G. 14,605

Dom G. $14, \mathrm{~S} 15$

Domange J.R. 14, S7

Domini L. $\underline{9}, 459, \underline{10}, 171, \underline{14}, 709$

Dominighini A. 14, 213

Donadieu A.-M. 12, 357

Donker A.J.M. $\underline{9}, 1017$

Donner M. $\underline{9}, 645,715,739,877, \underline{10}, 103, \underline{12}, 873$

Dörge L. 15,763

Dormandy J.A. $\underline{10}, 113,287,321, \underline{11}, 309$

Dörp E. 10, 375

Dosquet C. 12,817

Doucet D. 12,357

Douglas J.T. 12,867

Dougny M. 14, S53

Dreisbach A.W. 11, 295

Drew J.H. $\underline{9}, 375$

Driss F. $\underline{9}, 164, \underline{14}, 255$

Drouet L. 14,171

Drouin P. 12, 873

Droulle C. $\underline{9}, 162$ 
Duchesne-Delamaire M. $\underline{9}, 164,166$

Dufaux J. $\underline{9}, 797,14,83$

Dufourcq P. 14, 19, S1, 15, 627

Dugnani M. $\underline{9}, 654$

Dumont J.-M. 11, 465

Dundar S. $13,49,16,313$

Dupuy-Fons C. 15,191

Durand F. $\underline{9}, 164,166,458,460,625$

Durussel J.J. 9,797

Duwig I. 10,43

Ebel H. 10,637

Eber O. $\underline{9}, 328, \underline{12}, 9,93,15,649$

Eckhardt $\mathrm{H}$. 14,45

Edremitlioglu M. 14, 591, 15, 97

Egbrinkoude M.G.A. 12, 59

Egginton S. 12, 677

Ehringer $H . \underline{9}, 109,15,3$

Ehrly A.M. $\underline{9}, 461, \underline{10}, 473,625, \underline{12}, 41,223$, $14,263,16,3,249,319,321$

Eijzenbach V. $\underline{9}, 448,463$

Eimerl D. 10, 65

El Bouhmadi A. 14, 105, 637, 643

El-Gatit A. 12, 229

Elicio N. 11, 9

Elkeles R.S. $\underline{9}, 101$

Ellory J.C. $\underline{9}, 247,447,1009, \underline{10}, 535, \underline{14}, 545$

Elsner R. $\underline{12}, 657$

Elwood P.C. 12, 545

Erdincler D.S. 16, 271

Erdlenbruch K. 15, 601

Erdlenbruch W. 16,329

Ernst E. $10,157,525,11,107,129,303,429,575$ $\underline{12}, 35,521,549,805, \underline{15}, 867$

Ertan N.Z. 13, 473

Escolar G. 11, 339

Escousse A. 14, 663

Espírito Santo J. $\underline{9}, 648,649$

Eterovic D. 15, 841

Evans S.-A. 11, 79, 12, 255, 13, 73, 14, 149

Ewald U. $\underline{9}, 603$

Falke P. 11, 35

Famodu A.A. 15, 897

Fanari P. $\underline{9}, 439$

Fasching P. 15, 3

Faulborn J. 14, 45

Fazekas F. 14, 841

Fedele D. 11, 405

Fedele M.G. $\underline{9}, 999$

Fédou C. $9,351,953$

Feher M.D. 11, 441
Feher M.D. 9, 101

Ferdinand P. 12, 79

Fernandez A. 14, 321

Ferrara F. 16, 523

Ferreira D. $\underline{9}, 651,656$

Ferrone F.A. 12, 163

Fialon P. 15,627

Fidler V. $\underline{9}, 553$

Filippo de M. 9,656

Fioravanti P. 15,73

Fischer G. 13, 491

Fisher A.C. 12, 677,857

Fisher D. 10, 225

Fisher M. $\underline{9}, 441$

Fisher T.C. $10,263, \underline{12}, 109$

Fletcher J. 14,379

Fonk T. $\underline{9}, 1017,1029$

Fons C. $\underline{13}, 187,201,215,651, \underline{14}, 105$

Fontani R. 12, 697

Forbes C.D. 10, 263

Forconi S. $\underline{9}, 27,646, \underline{12}, 527,697, \underline{13}, 13$, $14,797, \underline{16}, 17,653$

Forman M.B. 12,121

Förster H. 14, 189

Fossat C. $9,163,11,649,12,67$

Francavilla G. 12, 449, 13, 227

Franchi M. 13, 177, 687

Franchin A. 11, 405

Francione $S$. 11,9

Franco de V. $\underline{9}, 459$

Franke A. 16, 249, 321

Franke R.P. 15,817

Frattini P.L. 11,325

Freidl W. 12, 705, 14, 841

Freyburger G. $\underline{9}, 161,181,450,451,871$

Friederichs E. $\underline{10}, 81, \underline{13}, 55$

Frigerio C. $\underline{9}, 459, \underline{10}, 171, \underline{13}, 177,687, \underline{14}, 709$

Frosi F. 13,215

Fujikawa M. $\underline{9}, 376, \underline{11}, 251$

Fukuuchi Y. 13, 253, 15, 89, 16, 143, 497

Fukuyama M. 13,623

Fumelli P. $\underline{9}, 450,653,654, \underline{11}, 641, \underline{15}, 73$, 16,543

Furesi L. $\underline{13}, 13$

Ga Tei 15,619

Gaal Van L. $\underline{9}, 983$

Gaehtgens P. 14, 421

Gal L. $\underline{9}, 209$

Galacteros F. 12, 415

Galanos C. 13,45

Galaris D. 15, 107

Galdo G. 14, 585

Galea G. 10, 21 
Galigani C. $\underline{9}, 459, \underline{10}, 171, \underline{11}, 361, \underline{13}, 177$

Gallo P. 9,999

Gamand S. 15, 167

Gambaro M. 13, 245

Gans R.O.B. $\underline{9}, 1017$

Gao Shi-Jia 9, 297

Gao Tie 16, 291

Garcia Cruz M. 9, 450

Garmo de P.L. 11, 167

Garnier J. 13, 481

Garnier M. $13,481, \underline{15}, 325$

Garosi G. 11, 41

Garry J. $\underline{9}, 166,458$

Gascoyne S.C. $12,627,639$

Gastpar H. $\underline{9}, 440$

Gaudey F. 14, 255

Gaudio R. 16, 653

Gavella M. $\underline{9}, 965, \underline{10}, 477,14,347$

Gavish B. 14, 497

Gelb A.W. 16, 533

Genetet B. $\underline{9}, 164,166,458,460,625, \underline{11}, 605$

Gennaro A.M. 16, 117

Gentile T. 14, 203

George S.J. 13, 661, 16, 479

Gerhardinger C. 11, 405

Gerlach A. $\underline{9}, 441$

Gevers Leuven J. $\underline{9}, 463$

Giansanti R. $\underline{9}, 450,653,654, \underline{11}, 641, \underline{15}, 73$, 16,543

Giavardi L. 10,541

Gilgenkrantz $\underline{9}, 219$

Gillespie J.A. 10, 241

Gioia D. $\underline{9}, 656, \underline{11}, 41$

Giovagnoni M.G. 16, 559

Giovannini M. 12,573

Girtanner C. 15,167

Glöckner W.M. 12,725

Gnasso A. 9,81

Goedhart P.T. $12,381, \underline{13}, 465, \underline{14}, 605,619$

Gogolwske I. 10, 297

Goll S. 10,43

Goncalves M.L. 9, 653

Gori S. 16, 57, 245

Goslinga H. $\underline{9}, 448,553,583,593, \underline{12}, 27,883$

Gotoh. F. 10, 515

Goudonnet H. 14, 663

Grandjean E.M. $\underline{10}, 215$

Grauer M.T. 16,367

Grebe R. $\underline{9}, 443$

Gribbon P.M. 13, 473

Grieshofer P. 14,841

Griffith B.P. 11,325

Grifo G. 11, 49, 617, 13, 227, 15, 53, 657

Grimaldi A. 9, 281

Gueguen-Duchesne M. $\underline{9}, 625$
Guerrini M. $\underline{9}, 27, \underline{12}, 527,697, \underline{13}, 13, \underline{14}, 797$, $16,17,57,245$

Guideri F. $\underline{9}, 459, \underline{10}, 171, \underline{11}, 361, \underline{13}, 177$, 15,367

Guillausseau P.J. $\underline{9}, 855,12,713$

Guillet R. $\underline{9}, 159, \underline{11}, 55, \underline{14}, 117,255$

Guillou J. 14, 83

Guimaraes J.P. $\underline{9}, 651,656$

Guiral V. 16, 235

Güler S. 11,497

Güner R. 11, 121

Guoyan J. 11, 183

Gupte R. 12, 427

Gupte R.V. 10, 95

Gustavsson C.G. 14, 677

Haag S. $\underline{9}, 452$

Haas A. 12, 17. 14, 45, 16, 367

Habon T. 14, 329, 15, 867

Haiyan Li 16, 603

Hall L.D. 9 , 446, 447

Hamai H. 12, 109, 733

Hamazaki T. $\underline{9}, 376, \underline{11}, 251$

Hammad A. 10, 225

Hammi H. 14, 117

Han Zhan-Shan 11, 459

Handa M. 13,623

Handt S. 12,725

Hansen Ch. $\underline{9}, 219$

Hara H. 12, 267

Hardeman M.R. 12, 381, 13, 465, 14, 605, 619, $16,11,151$

Haring H.-P. 14, 11

Haritou M. 14, 369

Harkness J. $\underline{9}, 35$

Harris A. 13,435

Harrison M.J.G. $\underline{9}, 139$

Hartert H. 10, 401

Hasegawa S. 14, 557, 571

Haung Y. 9 , 369

Haupt H. $\underline{9}, 125$

Haupt H. 452

Hawkey C. 12,639

Hawkey C.M. 12, 627

Hayashi K. $\underline{9}, 149$

Haywood L.J. 9, 923

Healy J.C. 15,167

Hebbel R.P. 12, 185

Hedner P. 13, 237, 791, 14, 831

Heethaar R.M. $9,453,14,769$

Heilmann L. $12,237, \underline{13}, 501,719,729$, $\frac{15}{15}, 311,359$

Heimburg P. $\underline{9}, 307$

Hein H. 10, 637 
Heins M. 15,737

Heitz J. $\underline{9}, 443, \underline{10}, 297, \underline{11}, 63$

Hell K. $\underline{9}, 329$

Hempelmann G. 12, 71

Hemrighausen A. $\underline{9}, 443$

Hennicke G. 12, 341

Henny Ch.P. 16, 533

Henrich H.A. $\underline{9}, 454,458, \underline{15}, 829$

Hepp K.D. 16, 623

Herrmann T. 15, 167

Heuvelmans J.H.A. $\underline{9}, 553,583,593, \underline{12}, 27$

Hildesheimer M. 10, 59

Hiramori K. 10, 435

Hiruma H. 11, 91

Hobbs J.B. $\underline{9}, 375$

Hoberg A. 11, 623

Höffkes H.G. $\underline{12}, 41, \underline{16}, 249,321$

Hoffmann E. $\underline{9}, 455$

Hoffmann J.J.M.L. 12, 217

Hoffmeister H.C.A. 15,335

Hombach V. 12, 549, 15,335

Hong Fan 16, 507

Hongo A.T. 14, 557

Horner S. 14, 841

Horng Sh.-SH. 15, 25

Horvath I. $\underline{14}, \underline{329}, \underline{15}, 867$

Horwitz E.R. 14, 545

Hosmane R.S. 15,877

How T.V. 12,45

Hu H.H. 14,723

Hu Q.H. 16, 645

Huang Ch.-R. 15, 25

Huang Yi Min $\underline{12}, 593$

Huang Youtian 10, 205

Hughes C.L. $\underline{9}, 442$

Huilgol N.G. $15,37,755$

Huisjes H.J. $\underline{9}, 553$

Huisman A. $\underline{9}, 553$

Hummel B. $\underline{16}, 611$

Hung T.-C. 11,325

Hunt W.B. 13,661

Hunt W.B. 16,479

Huntsmann A. 12, 357

Hussain M.A. $\underline{14}, 685, \underline{15}, 37,61, \underline{16}, 165$

Idée J.-M. 12, 357

Ihme N. 15, 681

lino M. 14,571

lijima S. $\underline{15}, 157$

Ikeda $Y, 13,623$

Ikemoto S. $\underline{9}, 371, \underline{10}, 463,11,175, \underline{12}, 935$, $14,509,16,713$

Imamura T. $\underline{10}, 215, \underline{11}, 465$

Inglese A. $\underline{9}$,
Ingram D.A. 12, 121

Intaglietta M. 11, 417

Irmler A. 14, 841

Isbister J.P. 14, 305

Isenberg D. 11, 501

Islam M.S. 13,261

Isogai, Y. $\underline{9}, 371, \underline{10}, 463,11,175,12,599,935$, $\underline{14}, 509, \underline{16}, 713$

Isushima N. $\underline{9}, 371$

Itagaki I. 13, 623

Ito M. 11,91

Itoga H. 15, 157

Itoh D. $\underline{15}, 89$

Izhong C. 12, 279

Jackson L.J. 12,947

Jaiswal V.S. 12,579

Janbon C. 15,191

Janot C. 10, 103

Janzen J. $\underline{9}, 695$

Jayaraman G. 15,715

Jeraldo T. $\underline{13}, \underline{519}$

Jiang Guo-vian $\underline{9}, 374$

Jiang Sh.-x. 15,859

Jiesheng Lu $\underline{16}, 603$

Johnn H. 12, 639

Johnson C.S. 12, 109

Jolly D. $\underline{9}, 739$

Jones J.G. $\underline{11}, 79, \underline{12}, 255, \underline{13}, 73, \underline{14}, 149$,

Jong J. $\underline{9}, \underline{448}$

$$
\frac{11}{15}, 877
$$

Judkiewicz L. 10, 561

Juge J. 15,167

Juhan-Vague I. $\underline{9}, 163, \underline{10}, 321, \underline{11}, 649, \underline{12}, 67$

Jung $F$. $11,623,657,12,437,557,725,747$,

$\frac{13}{68}, 93,435,14,189,355,565,15,601$,

Jung M. $\underline{9}, 109$ $681,707,817, \underline{16}, 329,343,611,631$

Jünger M. $14, S 65$

Juricskay I. $\underline{14}, 329, \underline{15}, 867, \underline{16}, 187$

Kaffarnik H. 15,737

Kaibara M. 14,509

Kakkar V.V. 10,339

Kaku Sh. 11, 351

Kale P.A. $\underline{10}, 329$

Kaleridis V. 14, 489

Kameneva M.V. 15,633

Kanakaraj P. $\underline{9}, 257$

Kandemir N. $\underline{9}, 577$

Kane O. 10,43

Kaneda M. $\underline{9}, 376,11,251$

Kaps H.-J. $\underline{9}, 462$

Kar S. $\underline{14}, 685, \underline{15}, 61, \underline{16}, 165$ 
Karakoc Y. 16, 271

Karakousis C. 9,440

Kässer U. $\underline{9}, 307$

Kasturiranga.7 S.P. 9,377

Kato T. $9,149,10,435$

Katoh S. 12, 297

Kaul D.K. 12, 191

Kawakami S. 14, 509

Kedaria M. 16, 533

Keeling D. 11, 501

Keil U. 12, 549

Kenner T. 10, 375

Kenner Th. $\underline{9}, 319$

Kent P. 14, 245

Kent P.J. 10,547

Keogh E. 13, 737

Kester R.C. 10, 547, 14, 245

Keuchel M. 1ㅁ, 637

Khalife K. $\underline{9}, 219$

Khan M.M. 15, 37, 755

Khodabandehlou T. $\underline{9}, 160, \underline{10}, 557, \underline{11}, 597$,

\section{$14,553, \underline{16}, 303$}

Khodabandehlou T. 16,677

Kiesewetter H. 11, 623, 657, 12, 437, 557, 747, $14,189,355,565,15,601,681$,

Kikuchi Y: 15,157 $707,817, \underline{16}, 329,343,631$

King R.G. $\underline{9}, 809$

Kitajima A. 12, 267

Kitao M. 12,297

Klaren J. 12, 455

Klee A. 13, 501

Kleinert G. 14, 37

Kleinschmidt S. 14,137

Klotz K.-F. 16, 487

Knabben H. 16, 357

Knox R.J. 9, 273, 11, 167, 12, 947

Kobari M. 10, 515, 15, 89, 16, 143, 497

Koch D. 16,367

Koch M. 14, 841

Koenig W. 12, 549, 15, 335

Koeppen S. 12, 237

Kollegger $H .10,499,14,339,16,285$

Költringer P. 9, 328, 12, 9, 93, 15, 649

Konno K. 13, 23

Konno S. 16, 143

Kooshesh F. 11, 79

Koppensteiner R. 9,109, 15, 3

Korantzopoulos P. 15,107

Kormos R.L. 11, 325

Koscielny J. $14,189, \mathrm{~S} 65,15,601,707,817$, $16,329,343,631$

Koutsouris D. $\underline{9}, 451, \underline{10}, 43, \underline{11}, 55, \underline{14}, 369$,

Kovacs B. $16, \frac{15}{187}$
Kowal P. 13, 701

Kraegen E.W. 9, 377

Krause S. 16, 343

Kriechbaum A. 15, 359

Kröll W. 12, 79

Krotkiewski M. 15,641

Krüger W. $\underline{9}, 442$

Ktorza A. 14, 83

Kübler W. 10,601

Kuchiba K. $\underline{9}, 371,11,175,12,935$

Kudrimoti H.S. 14, 321

Kulkarni M.V. 14, 271

Kumaravel M. $\underline{15}, 273,291$

Kuppe H. 16, 487

Kuriyama Y. $\underline{9}, 297$

Kurzemann S. 15,3

Kutman N.N. 9,577

La Terra G. 9,654

Laan de Vries van der, E. 12, 27

Labios M. 16, 235

Laborde J.C. 15,191

Labrouche S. 9, 451

Lacombe C. $\underline{9}, 160,165,647, \underline{11}, 387$, $12,415,511,733$

Ladjouzi J. $\underline{9}, 160, \underline{11}, 387, \underline{12}, 415,733$

Laffargue F. 14,643

Laffranchi M. 11, 447

Lageweg E. 9,448

Laghi Pasini F. $\underline{9}, 459, \underline{10}, 171, \underline{13}, 177,687$,

Lagleyze P. $\underline{9}, 376$ $14,709,15,221,367$

Lagoueyte C. 9,953

Lagrasta C. 11,605

Lakomek M. 13, 55

Landgraf $\mathrm{H} . \underline{14}, 263, \underline{16}, 249,321$

Landgraf R. 16, 623

Lane I. 13, 73

Lang P.D. 13,679

Lange $H .10,637$

Langer R. 15,829

Langsteger W. 12, 9, 93, 15, 649

Lapolla A. 11, 405

Larsson $\mathrm{H}$. $\underline{10}, 321,11,317, \underline{13}, 237,14,677$

Lartigue B. 13, 779

Launois B. $9,166,458$

Laustriat D. $10,43,11,583$

Lavalle $\mathrm{Ph} . \underline{16}, 35$

Lawall H. 9,441

Layoueyte C. 9,166

Le Beyec J. 15, 667

Le Blanc M. 12, 733

Le Dévéhat C. $9,161,162,164,237,543,845$, $861, \underline{10}, 557, \underline{11}, 597, \underline{14}, \mathrm{~S} 53$, 


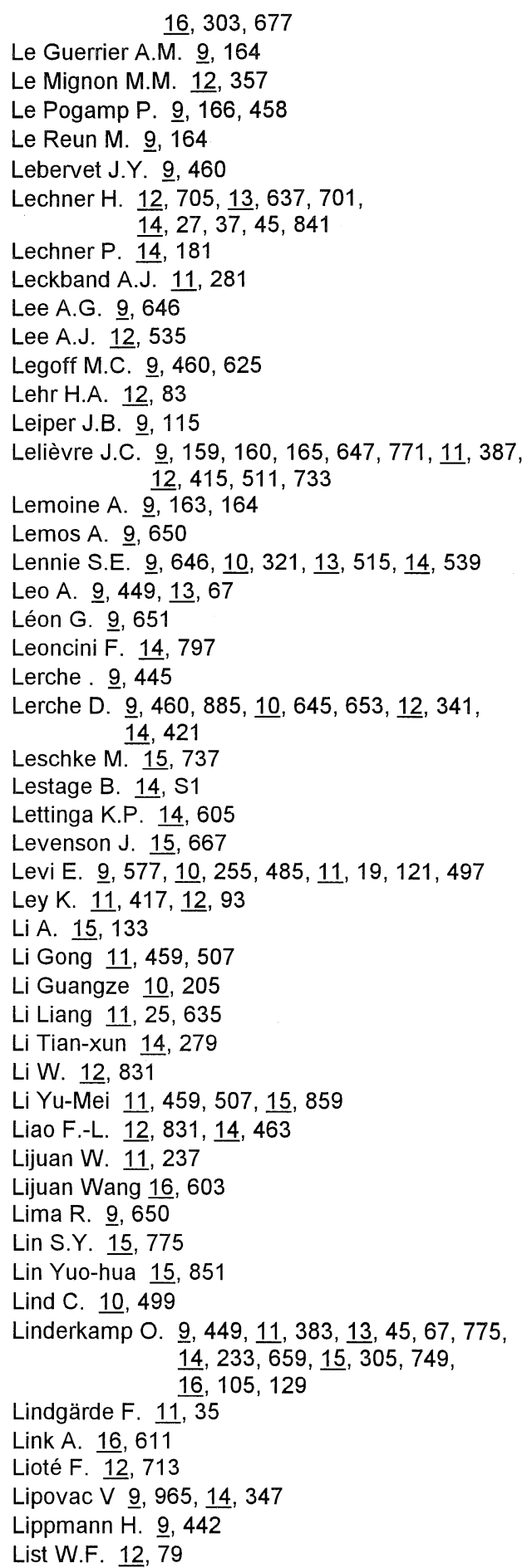

Liu Bo 15,641

Llopis I. $\underline{15}, 677, \underline{16}, 235$

Lo Presti R. $9,373,455,456,10,449,11,1,49$,

Lo Y.K. 14, 723 $617,12,449,13,35,227,14,393$, $597, \underline{15}, 53,657,16,177,523$

Lombardini R. 9,457

Long Mian $\underline{16}, 135$

Longhini E. $\underline{9}, 89,439,823, \underline{16}, 559$

Longshao Xu 12, 279

Longy-Boursier M. 15, 627

Loosemore T. 11, 309

Lopovac V. 10, 477

Loukopoulos D. 10, 35

Loureiro J. 9,651

Lowe G.D.O. $\underline{9}, 644,646, \underline{10}, 321, \underline{12}, 535,757$, $867,13,515, \underline{14}, 539$

Lowe K.C. 12,141

Lu Chuan-zheng 10, 309

Lu Shu-Hua 11, 459, 507

Lu Zhi-h. 15, 859

Lucas G.S. 10, 35

Luk Y.O. 14,723

Luo Guang $\underline{9}, 615$

Lupattelli G. $\underline{9}, 457$

Luquita A. 14, 213, 16, 117

Ma Wei-yuan 14, 279, 15, 81, 619, 859, 트, 291

MacRury S.M. 12, 757, 14, 539

Madan M. 11, 295

Maeda N. 14, 53

Maeda T. $\underline{9} ; 144,480,533, \underline{12}, 857,935, \underline{14}, 509$

Mahafzah M. 10, 225

Mahler Y. 14, 497

Maignan M.-F. 10, 231

Maleßa R. 12, 237

Mallard C. $13,187,201,651$

Malone J.I. 14, 481

Manasse G. 16, 57, 245, 653

Manciet G. $\underline{9}, 450$

Mannarino E. 9, 457

Mar Velert Ma. 16, 549

Maragoni G. $\underline{9}, 457$

Maraninchi D. 12,67

Marc B. 15,325

Marenco S. $11,9, \underline{13}, 245$

Mari A. $\underline{9}, 369, \underline{14}, 413$

Marinova T. 15, 45

Marotta G. 13,13

Marrali F. $\underline{9}, 633, \underline{11}, 447$

Marschall M. 11,129

Martin G. $\underline{9}, 41, \underline{12}, 369,761$

Martínez M. $11,133, \underline{13}, 447, \underline{14}, 419, \underline{15}, 677$, $\underline{16}, 235,277,515,549$ 
Martinot F. 10, 231

Martins e Silva J. $9,641,647,648,649,650$, 651,656

Maruyama S. 13,623

Marx U. 14, 565

Mary A. 9, 209, 439, 12, 287

Masahiko Seike $\underline{9}, 909$

Mascioli A. 14, 203

Massad L. $\underline{9}, 339$

Materazzi M. 10, 171

Matos L. $\underline{9}, 647$

Matrai A. 11, 575

Matsuda T. 10, 435

Matsuo H. 10, 435

Matsuoka S. 13,253

Maughan R.J. $\underline{9}, 115,10,51$

Maurer A. $\underline{9}, 443$

Maurer U. 16, 611

Mavromatis G. 10,43

Mayer W.-K. $\underline{9}, 442$

Mazoyer E. 14, 171

Mchedlishvili G. $14,285,15,697,783, \underline{16}, 193$

McKie K.M. 13,519

McKie V.C. $12,587,13,519$

McLver M.A. $\underline{9}, 375$

McMillan D.E. $\underline{9}, 791,989,12,787,13,147$,

Meffert H. 9,444

$14,473,481, \underline{16}, 669$

Meier C. 16,631

Meier W. $\underline{9}, 885, \underline{10}, 653$

Meiselınan H.J. $\underline{9}, 1,55,441,446,723,923,935$,

Mela-Riker L. 9,273 $2,109,657,841,13,575$

Meli F. 16, 523

Melis V.M.J. 12, 27

Mellinghoff A.C. 16,623

Menger M.D. 12,83

Merchant R. 14, 321

Mercié $P$. 15, 627

Mercuri M. $\underline{9}, 457$

Messa G.L. $\underline{9}, 459, \underline{10}, 171,13,177$

Messmer K. $10,383,12,83$

Meyer M.S. 14, 401

Meyer R. 10, 191

Mezey B. 14, 329

Miao Ge 16,267

Miao H. $\underline{15}, 851, \underline{16}, 645$

Micallef J.P. $14,807,15,13,201,213$

Michaelis P. 9,444

Miguel M.J. $\underline{9}, 652$

Mihara B. 10,515

Milani S. 11,447

Miller H. 9, 445, 10, 645

Miller V.M. 14, S37

Mills P. $9,281,715$
Minar E. $\underline{9}, 109$

Mineo F. 11, 605

Mira Y. 16,277

Mirshahi M. 11, 649

Missirlis Y. $13,767, \underline{14}, 489$

Moatti N. 15,667

Moccia G. 11, 605

Möckel A. $\underline{9}, 445$

Möckel C. 10,645

Modat G. $\underline{9}, 209, \underline{12}, 287$

Modesti P.A. 16, 701

Mojiminiyi F.B.O. 14,545

Mokken F.CH. 16, 533

Molina A. 14, 585, 16, 229

Molina E. 10,185

Molina Font J.A. 16, 229

Molina J.A. 14,75

Moll A. 16, 343, 631

Momatselidze N. 15,783

Mombelloni A. 10,185

Monaci A. $\underline{9}, 459,10,171$

Monnier J.F. 15, 147

Montana M. $12,449,13,227,14,393,597$

Montefusco S. $\underline{9}, 81$ $15,53,657, \underline{16}, 177,523$

Moreira C. $\underline{9}, 647,648,649,651,656$

Moreno V. $\underline{14}, 419$

Moser K. 9,569

Moser M. 10,375

Motta C. $\underline{9}, 47$

Mounie J. 14, 663

Mozsik Gy. 14, 329, 15, 867

Mraz W. 12, 549

Mrowietz C. $\underline{12}, 747, \underline{14}, 189, \underline{15}, 707, \underline{16}, 343$

Muchnik Ch. 10, 59

Mukherjee M. 13, 759, 14, 127, 131, 223

Müller G. 16, 487

Müller G.H. 16, 63

Muller S. $\underline{9}, 739, \underline{10}, 103, \underline{12}, 873$

Munoz A. 14, 75, 585, 16, 229

Muralidharan E. $\underline{9}, 377, \underline{11}, 205$

Murata T. 14, 519

Murray J.J. 12, 121

Naga R. $9,371,11,227$

Nagai A. 13,23

Nagakawa $Y .11,351$

Nagel R.L. 10,35

Nain-dit-Ducret M. 12, 357

Nakamura T. $\underline{9}, 149, \underline{14}, 557$

Nalpas B. $\underline{9}, 451,11,55,14,255$

Nannmark U. 11, 369

Nappi G. $\underline{9}, 81$

Nara M. $\underline{10}, 515$ 
Narbona E. $\underline{14}, 75, \underline{16}, 229$

Nash G.B. $10,113,275,287,321,353,535$, $11,309,12,57,109,677,857,13,407$ Nasrawi F. $\underline{9}, 439,16,559$

Navarro-Prigent M.J. 14, 819

Neher G. 16, 611

Neumann F.-J. 모 125, 452, 10, 601, 13, 679

Neumann S. 13,501

Neves D. $\underline{9}, 652$

Nguyen P. $\underline{9}, 162, \underline{13}, 155, \underline{14}, 739$

Nguyen $\mathrm{Ph} .13,779$

Nicolas P. 13,481

Niederkorn K. 14, 841

NiiMi H. 16,645

Niimi, H. $\underline{9}, 297$

Nijhof E.J. $\underline{14}, 769, \underline{15}, 803, \underline{16}, 151$

Ning T. 11, 237

Ning Tang 16,603

Nobili F. $11,9, \underline{13}, 245$

Nobre A. $\underline{9}, 650$

Nobuji Maeda $\underline{9}, 909$

Noe A. $\underline{9}, 452$

Nolte D. 12,83

Nomura T. 14,571

Norcliffe D. $10,165,491,12,767$

Nordt F.J. $\underline{9}, 273,374,11,167$

Norris S. $\underline{9}, 923$

North M.L. $\underline{10}, 43, \underline{11}, 583$

Nunes M. $\underline{9}, 650$

O'Brien J.R. $\underline{9}, 331, \underline{12}, 545$

O'Rear E.A. 10, 505

Oberndorfer S. 16,285

Oder B. $10,499,14,339$

Oder W. $10,499, \underline{14}, 339, \underline{16}, 285$

Ogawa J. 11,175

Ohba H. $\underline{15}, 157$

Ohta K. $\underline{10}, 515$

Ojeh E.A. 15, 897

Olivetti G. 11,605

Önen S. 16, 271

Oratore A. $\underline{9}, 999$

Orimo H. 11,351

Orsetti A. $\underline{9}, 166,351,361,953,13,187,201$ $215,651, \underline{14}, 105,643,807, \underline{15}, 13$, $147,191,201,213$

Osterman G. $\underline{9}, 165$

Othmane A. $\underline{9}, 281,797, \underline{14}, 83$

Otsuka F. $\underline{15}, 157$

Oviasu E. $\underline{15}, 897$

Ozgen T. $\underline{13}, 459$

Paganardi L. $\underline{9}, 655, \underline{10}, 541, \underline{12}, 573 \underline{10}$

Pagliano L. $\underline{9}, 823$
Palareti G. $\underline{10}, 321$

Palazzetti D. $\underline{9}, 457$

Palm ST. 15, 641

Palmer A.A. 9,375

Paltriccia R. $\underline{9}, 457$

Pandolfi G. $\underline{9}, 81$

Panno A.V. 13,83

Papies B. 10, 645

Parfenov A.S. 15,633

Paries P. 15, 325

Parnetto L. $\underline{9}, 457$

Parreira F. $\underline{9}, 652$

Pasini F.L. 11, 361

Pasqui A.L. 11, 361

Passaretti B. $\underline{9}, 655$

Pastorelli M. 10, 171

Paterson K.R. 14, 539

Patterson K. 11, 501

Paul J.-L. 15,667

Paulitschke M. $\underline{9}, 885, \underline{10}, 653, \underline{13}, 407$

Paulsen H.F. 11, 107

Pautou C. 14, 255

Pearce J.M.F. 10, 113

Pearson M.J. 13, 473, 14, 531

Pecchi S. $\underline{9}, 459, \underline{10}, 171, \underline{14}, 709$

Pellegrin J.L. 15, 627

Pellerin F. 15,191

Perret G. 13,481

Perrotin P. $\underline{9}, 451, \underline{11}, 55, \underline{14}, 117,255$

Persson S. 10, 321

Persson S.U. $11,317,13,237,791$,

$$
14,677,731,831
$$

Persson Stig 14,677

Pesch T.W. 12, 437

Petitfrère E. $\underline{9}, 739, \underline{13}, 155$

Petri S. 15, 221

Pettit K.L. $13,661,16,479$

Pfafferott C. $\underline{9}, 55,370, \underline{10}, 423, \underline{12}, 841$

Pfitzner D. 10, 637

Phadke S. 14, 321

Phat D. 11, 417

Phipps C. 12, 639

Picciolini F. 14, 797

Picone F.P. 11,49

Pieragalli D. $\underline{9}, 27,459, \underline{10}, 171, \underline{11}, 361$, $\underline{13}, 177, \underline{15}, 367$

Pierer G. 12, 9,93

Pietsch L. $\underline{9}, 723$

Pignon B. $\underline{9}, 739, \underline{14}, 739$

Pikon A. 12, 357

Pina Cabral J. $\underline{9}, 652$

Pindur G. $\underline{12}, 557,747, \underline{13}, 93, \underline{14}, 189, \underline{15}, 601$, 16,631

Pintaric I. $\underline{15}, 841$

Pinto Correia J. $\underline{9}, 647$ 
Pinto Y. $\underline{9}, 647,648$

Player M. $\underline{9}, 1009$

Plettig J. $\underline{9}, 444$

Plotkine M. $\underline{9}, 339$

Poggi M. 10, 321

Pogglitsch H. 10, 375

Pohl P. 14, 11

Pollari G. $9,369,439$

Pollock J.G. 12, 867, 13, 515

Pölz W. 14, 11

Pommereuil M. $\underline{9}, 625$

Ponari O. 10,185

Porta P. $\underline{9}, 1023$

Pöschl J.M.B. $13,45, \underline{15}, 749, \underline{16}, 129$

Postiglione A. $\underline{9}, 81$

Potron G. $\underline{9}, 162,165,739, \underline{13}, 155,779, \underline{14}, 739$

Prata M.M. $\underline{9}, 649$

Prencipe M. 9, 999

Provvedi T. $\underline{13}, 177,687, \underline{14}, 709, \underline{15}, 221,367$

Pruvost A. $14, \mathrm{S1}, 15,627$

Puertas A. 14,75

Puniyani R.R. $\underline{9}, 199,373, \underline{10}, 95,329, \underline{11}, 243$, $397,12,427,579,14,271,321$ $685, \underline{15}, 37,61,347,755, \underline{16}, 165$

Qi Ying 10, 205

Qian M. Q. 12,593

Qiu Guoyong 16, 267

Quaini F. 11, 605

Quemada D. $\underline{9}, 781$

Quin Fu-Z. 14, 779

Quing Zhao 11, 183

Quing Zhi-jiu 10, 309

Quintao T. $\underline{9}, 648,649,650$

Raasch W. 16, 487

Rabetafika E. 14, 819

Rabini R.A. $15,73, \underline{16}, 543$

Radberg G. 12, 229

Rädisch T. 10, 81

Radtke H. $14,355,15,707,817, \underline{16}, 343$

Rahfeld J. 12, 747

Rainaeri A. $\underline{9}, 455$

Rainer C. $\underline{9}, 923$

Rainer F. $\underline{9}, 319$

Raininko R. 12,393

Rama D. 15, 201

Rampling M.W. $\underline{9}, 41,101,442, \underline{10}, 339, \underline{11}, 441$, $12,369,639,76113,473$,

Ramschak H. 12,79 $\underline{14}, 93,531,697, \underline{16}, 23$

Ranade G.G. $15,37,755$

Raneli G. 13,83
Ranieri R. $\underline{9}, 655, \underline{10}, 541, \underline{12}, 573$

Rao P.R. 15,877

Rao S. 14,321

Rapin D.R. 13, 261

Rapin J.R. 14,663

Rasadini 11, 9

Rasia M. 14, 213, 16, 117

Rasia R.J. 9, 1023, 14, 213, 15, 177, 687

Rauturier M. $\underline{9}, 166$

Razzavian M. 16, 303

Razzavian S.M. 15, 667

Reid A.W. 13, 515

Reim M. $\underline{11}, 623, \underline{12}, 437,725, \underline{13}, 435, \underline{16}, 357$

Reiner Z. 15, 841

Reinhart B. 14,841

Reinhart W.H. 13, 491

Reininger A.J. $\underline{16}, 623$

Reisecker G. 12,9

Reneman R.S. 12,59

Resch K.L. 11, 107

Rewald E. $\underline{9}, 1023$

Ribeiro C. $\underline{9}, 651,656$

Ribeiro J.A. $\underline{9}, 653$

Ribier F. 14, 255

Ribitsch V. $\underline{9}, 313$

Ricci R. 11, 605

Ricciardi G. $\underline{9}, 823$

Ricciotti R. $\underline{9}, 450$

Richmond W. $\underline{9}, 442$

Rieger H. 10, 321, 11, 479

Rifkind J. 15, 877

Rifle G. 14, 663

Righi G.A. 16, 245

Riha P. 16, 43

Rillaerts E. $\underline{9}, 983$

Rimmer A.R. 10, 263

Ripoll L. 14,171

Riquelme B. 15, 687

Roberts D. $12,229,15,641$

Robertson J.D. $\underline{9}, 115, \underline{10}, 51$

Robinson R.J. 11, 441

Roche M. $\underline{9}, 47$

Rodeck C.H. 14, 93

Rodrigues M. $\underline{9}, 648,649$

Rodriguez G. 11, 9, 13, 245

Roll G. 11, 63

Roll, D. $\underline{9}, 443$

Romagnoli F. 15, 73

Romandini St. 12, 449

Romano A. $\underline{9}, 455, \underline{13}, 227, \underline{15}, 657$

Romanoff N. $\underline{9}, 989$

Rosadini G. 13,245

Rosasco García M. 모 1023

Rossmanith K. 15,829

Rother T. 15, 707 
Roudaut M.F. $\underline{9}, 161, \underline{14}, 19$

Rousseau O. 14, 637, 643

Rousset B. $\underline{9}, 163$

Roux J. 10, 215, 231, 11, 465

Rubba P. $\underline{9}, 81$

Rubinjoni Z. 10, 477

Rubinstein A. $\underline{9}, 372$

Rubinstein de Salama A. $\underline{9}, 559$

Rubinstein M. 10, 59

Rud K. 14, S37

Ruef P. $11,383, \underline{14}, 233,659, \underline{15}, 749, \underline{16}, 129$

Ruiz C. $14,585,16,229$

Rumley A. $12,535, \underline{13}, 515$

Rusch P. 15, 167

Sabio H. 12,587

Sabio T. 13, 519

Saeger-Lorenz K. 12, 223, 16, 249

Sagmeister $\mathrm{CH}$. 10, 499

Sahm U. 15, 133

Saihan E.M. 14, 379

Sainty D. 12,67

Sakanishi A. 15,889

Salama Benarroch L. $\underline{9}, 559$

Saldanha C. $9,647,648,649,650$

Saletti M. $\underline{9}, \underline{459}, \underline{10}, 171, \underline{11}, 361, \underline{13}, 177$

Salmon G.P. 9,331

Samama M. $\underline{9}, 456, \underline{12}, 455$

Samama M.M. 15,381

Sanchez R. $14, \overline{75}$

Sandhagen B. $\underline{9}, 603$

Sani P. 13,13

Saniabadi A.R. 10, 263

Santambrogio L. $\underline{9}, 457$

Santona L. 14,663

Santos A.I. $\underline{9}, 651,656$

Santos T. 15,677

Saradeth T. $10,157,525,11,107,303,429$, $\underline{12}, 521$

Sarcinella R. 14, 413

Sarna A. $9,373,455,455,456,839,10.449$,

Sartori M. $\underline{9}, 654$

$11,1,49,617,12,449,13,35,227$, $14,393,597,15,53,657$

Savakis M. 13,767

Savitsky J.Ph. 10,225

Sawada T. $\underline{9}, 297$

Sawazaki S. $\underline{9}, 376, \underline{11}, 251$

Sayyid S. $\underline{9}, 440$

Scaglioni S. 12,573

Scarpato N. 9,81

Schaaf P. 16,35

Schabner W. $\underline{9}, 441$

Schäfer J.H. $\underline{\underline{9}}, 443, \underline{10}, 297, \underline{11}, 63$
Schärer K. $\underline{9}, 449, \underline{13}, 67$

Schechter A.N. 14, 557, 571

Scheffler A. 10, 321, 11, 479

Schenk J. $\underline{9}, 462$

Scherf H.P. $\underline{9}, 444,445, \underline{10}, 645$

Schernberg F. $\underline{9}, 165$

Schichl K. 14, 421

Schick P. 15, 335

Schied G. 14,37

Schieffer H. 11, 657

Schieffer H. 16, 611

Schiffer H. 16, 343

Schikarski C. 13,529

Schimetta W. 14, 11, 189

Schimetta W. 16, 631

Schimrigk K. 16,367

Schlappritzi E. 13, 491

Schlierf G. 13,679

Schmid-Schönbein H. $\underline{9}, 443,444, \underline{12}, 883$,

Schmidlechner $\mathrm{CH} .11,107$

\section{$13,803,16,63$}

Schmidt G. $9,460,10,653$

Schmidt R. 14,841

Schmitt Y. 12, 243

Schmucker $P$. 16, 487

Schnalzer E.A. 9,69

Schnauffer M. $\underline{15}, 749, \underline{16}, 129$

Schneditz D. $9,319,10,375$

Schneider H. 12,243

Schneider R. 12, 1

Schoebel F.C. 15, 737

Schooneman F. $\underline{9}, 877$

Schoute E. $\underline{9}, 463$

Schpitz-Droz R. 11, 387

Schröder R. $\underline{9}, 443, \underline{10}, 297, \underline{11}, 63$

Schroeder M. 10, 525

Schröter W. 13,55

Schulte K. 12, 437, 725

Schulte-Huermann D. 14, 263

Schut N.H. 13, 46514, 141, 619

Schwartz R.W. 13, 671

Schwendemann G. 12, 237

Schwerdt H. 16, 611

Seaman G.V.F. $\underline{9}, 273,367,374, \underline{11}, 167, \underline{12}, 947$

Seiberth V. 15,305

Seiffge D. 13,501

Seigneur M. 14, 19, S1, 15, 627

Sekkad M. $\underline{9}, 166,953$

Selinger W. $\underline{9}, 452$

Semple C.G. 14,539

Senger B. 16,35

Serjeant G.R. 10, 35

Serra A. $\underline{9}, 373,455,456,839, \underline{10}, 449,11,1,41$, $617, \underline{12}, 449, \underline{13}, 35,227, \underline{16}, 177$

Seshadri V. 15,715 
Setiadi H. 12,713

Sever P.S. $\underline{9}, 101,11,441$

Seyfert U.T. 12, 747, 14, 137

Shakarishvili R. 15, 783

Shand B.I. 9,370

Shapiro Y. $\underline{9}, 650,651$

Shaw S.J. $\underline{9}, 935$

Shen Qu 16, 507

Shi Y.D. 16,559

Shi Yen 15, 859

Shi Yong-de 10, 309

Shiga T. 14, 53

Shimizu T. 9 , 297

Shinohara T. 10, 515

Shio H. 11, 91, 14, 557, 571

Shu Chien $\underline{9}, 69$

Siadat M. $\underline{9}, 739$

Siepi D. $\underline{9}, 457$

Siewert H. $\underline{9}, 444$

Sigola L.B. 13,737

Silber R. $\underline{9}, 454$

Simon A. $\underline{15}, 667$

Simon E. 15,667

Singh M. $\underline{9}, 257,377, \underline{11}, 205, \underline{15}, 273,291$

Sirs J.A. $\underline{9}, 372,11,191$

Sixma J.J. $\underline{9}, 452,453, \underline{14}, 769, \underline{15}, 803, \underline{16}, 151$

Slaaf D.W. 12,59

Slonim A. $\underline{9}, 650,651$

Small M. 12, 757

Smith B.D. 10,241

Smith W.C. $\underline{9}, 646$

Smith W.C.S. $12,535,867$

Smolle-Jüttner F.M. 10 , 375

Snabre P. $\underline{9}, 281$

Sochor G.E. 14,45

Sodi N. 14, 709

Soler C. 15,167

Solves P. $16,277,515$

Somazzi R. $\underline{9}, 89,439, \underline{16}, 559$

Sonar S. 11,397

Sönnichsen N. $\underline{9}, 444$

Soria C. 11,649

Soria J. 11,649

Sowemimo-Coker S.O. $\underline{9}, 723$

Spatt J. 14, 339

Specht-Leible N. 13, 679

Spitzer S. $11,657, \underline{12}, 747$

Squeri M. 10,185

Stadler S. $\underline{9}, 440, \underline{10}, 225$

Stavenow L. 11,35

Steel S.A. 10, 113

Stegmeier K. 15,763

Steigerwald R. $\underline{9}, 454,458$

Stein D. 15,737
Steinbach U. 11,479

Steinmetz A. 15, 737

Stocker K. 12, 747

Stoll M. 12, 17, 16, 367

Stoltz J.-F. $\underline{9}, 219,645,645,715,739,877$ $\underline{10}, 103,12,873, \underline{14}, 237,789$, $16,1,35,43,87$

Stone P.C.W. $\underline{\underline{W}}, 181,247,897, \underline{10}, 275,535$, 11,113

Stoppa A.M. $1 \underline{12}, 67$

Storlien L.H. $\underline{9}, 377$

Strack van Schijndel R.J.M. $\underline{9}, 1029$

Strangfeld D. $\underline{9}, 444$

Strano A. 13,83

Strauer B.E. 15, 737

Streckstra G.J. 14, 769

Streiff F. $\underline{9}, 877, \underline{10}, 103$

Strickland N.H. 12,369

Strigberger J. $\underline{9}, 989$

Stringer M.D. $\underline{9}, 442, \underline{10}, 329$

Stuart J. $\underline{9}, 181,247,447,644,897,1009$ $\underline{10}, 35,275,535,11,113,663, \underline{12}, 109$, $203,309,407,797,13,3,571,743$, 14,545

Stubinski B.M. 13,245

Sugihara K. 12,185

Sukchut B. 15,729

Sukyasyan A. 16, 271

Sun Da-g. 15, 81, 859

Sun Da-gong 14, 279, 15, 619

Sun Z. 15,877

Sund M. 12, 549

Supparo I. $\underline{13}, 187,201,215,651,14,105$, $15,201,213$

Sushu Guo 16, 507

Suthisai N. 15,729

Swank R.L. 11, 281, 12, 947

Sweetnam P.M. $\underline{12}, 545$

Tahta K. 10,255

Takamizawa K. $\underline{9}, 149$

Takao M. $16,143,497$

Takashi Nakajima $\underline{9}, 909$

Takeda H. $\underline{13}, 253, \underline{15}, 89, \underline{16}, 143,497$

Takeshi Shiga $\underline{9}, 909$

Taki H. $\underline{9}, 376, \underline{11}, 251$

Talbert D.G. 14, 93

Tamao $Y \underline{12}, 267$

Tan Y. $\underline{9}, 273$

Tanahashi N. $\underline{10}, 515, \underline{13}, 253, \underline{15}, 89$,

Tanaka H. 16,, 16

$$
\frac{16}{713}, 143,497
$$

Tang Ning 10, 205

Tang Zheng-shen 10, 309

Tangelder G.J. 12 12,59 
Tao Zu-Lai 11, 459, 507, 14, 279

Tardio S. 10,185

Teitel P. $9,443,751$

Teixeira F. $\underline{9}, 649$

Temiz A. 14,591

Tempelhoff v. G.-F. 13, 729, 15, 311, 359

Terayama Y. 10,515

Thao Chan M. $\underline{9}, 47$

Thomas J.L. 10, 241

Thomas P.R.S. 11,309

Ticozzelli P. 16,559

Tien-Hsi Young 10, 191

Tillmann W. $10,81,13,55$

Tillmanns H. $\underline{9}, 125,452, \underline{10}, 601$

Tocilj J. 15,841

Tomasso De G. $\underline{9}, 653,654$

Tomita M. $\underline{10}, 515, \underline{13}, 253, \underline{15}, 89, \underline{16}, 143,497$

Tommaso de G. $\underline{9}, 450$

Tonelli C. 10,185

Toplan S. 16,271

Tornaghi G. $\underline{9}, 655, \underline{10}, 541, \underline{12}, 573$

Torregiani F. 15,687

Tortorolo U. 11,9

Toth K. $\underline{14}, 329,15,867, \underline{16}, 187$

Tozzi E. $\underline{9}, 999,14,203$

Tozzi-Ciancarelli M.G. 9 , 999, 14, 203

Trabalzini L. $12,697, \underline{13}, 13, \underline{14}, 797$

Traina M. $\underline{9}, 455$

Treib J. 12, 17, 16, 367

Troiani-Sevi E. $\underline{9}, 999$

Trouet A. 14, S15

Truchot R.C. 14, 663

Tsinamdzvrishvili B. 15,697

Tsushima N. $\underline{9}, 149, \underline{10}, 435, \underline{11}, 217$

Tunstall-Pedoe D.A. 12,505

Tunstall-Pedoe H. $\underline{9}, \underline{646}, \underline{12}, 535$

Turchetti V. $12,527,14,797$

Tuvemo T. $\underline{9}, 603$

Uberos J. 14, 75, 585, 16, 229

Ücer O. $\underline{9}, 577, \underline{11}, 121$

Uchiyama S. 13,623

Uhlich D. 15,737

Umansky-Zeverin M. 15, 687

Urakaza M. $\underline{9}, 376$

Urakaze M. 11, 251

Utterback N.G. $\underline{9}, 989$

Uyesaka N. 11, $91, \underline{14}, 557,571$

Vaccaro F. 11, 49

Vaden I, A, 11, 281

Vague P. 11, 649

Valensi P. 15, 325
Valenzuela A. $14,75,585, \underline{16}, 229$

Valetitsch H. 14, 27

Vallés J. 15, 677

Valverde de Rasis J. $\underline{9}, 1023$

Van der Werf A.J.M. $\underline{\underline{9}}, 463$

Van Voorthuizen H. $\underline{9}, 448$

Vandewalle $\mathrm{H} . \underline{12}, 415,511$

Vansant G. $\underline{9}, 983$

Varela G. $\underline{9}, 651$

Vater S. 13, 501

Vattimo A. 12, 697

Vayá A. $13,447,14,419, \underline{15}, 677, \underline{16}, 31,235$, $277,515,549$

Vekasi J. 16,187

Velcheva I. 15, 45

Ventimiglia G. 14, 393, 15, 53, 657, 16, 523

Verga S. $\underline{9}, 456$

Verwey W. $\underline{9}, 448$

Vessel B. $\underline{9}, 739$

Viala J.L. $\underline{13}, 215, \underline{14}, 643, \underline{15}, 13$

Viale P.A. 11, 9

Viana-Queiroz M. $\underline{9}, 648,649$

Vicariot M. 13, 261

Vicente O. $\underline{9}, 652$

Villa S. 16,559

Vimeux M. $\underline{9}, 164,237,543,845,861, \underline{14}$, S53

Vimeux M. 16, 677

Voegel J.-C. 16, 35

Voerman H.J. $\underline{9}, 1029$

Voisin Ph. $\underline{9}, 219$

Volger E. $\underline{9}, 55, \underline{10}, 423$

Volger $F . \underline{9}, 370$

Völkl A. 15,763

Volpi L. 14,709

Voutay M. 15,167

Waart van der F.J.M. 16, 533

Wachter Ch. J. 11, 325

Wagner B. 15,3

Wagner S. 15,305

Waldhäus। W. 15,3

Walitza E. $\underline{9}, 307,329, \underline{10}, 363, \underline{12}, 243$

Walker K.A. 10,51

Walzl B. $12,705, \underline{13}, 637,701, \underline{14}, 27,37,45,181$

Walzl M. $12,705, \underline{13}, 637, \underline{14}, 27,37,45,181$

Wang Baisheng $\underline{9}, 369, \underline{10}, 205$

Wang Hong-ru $11,459,507$

Wang Lijuan $\underline{9}, 369$

Wang $X .14,237,789$

Wardrop C.A.J. 11, 79, $\underline{13}, 73$

Watts E. 12, 499

Waugh R.E. 12,649

Wautier J.L. $\underline{9}, 855, \underline{12}, 55,713,817, \underline{14}, 63$,

$\underline{16}, 575,661$

Wautier M.P. $\underline{9}, 855, \underline{12}, 713, \underline{16}, 661$ 
Webb A.R. 10,287

Wei Xu 16, 507

Weidler B. $\underline{9}, 454$

Weill D. 12, 713

Weimer B. 11, 575

Weiss Th. 11, 383

Weitz G. 16, 487

Welch C.R. 14, 93

Wen Z.-y. 15, 81, 619, 859, 16, 291

Wen Zong-Yao 11, 459, 507, 14, 279

Wenby R.B. $\underline{9}, 1,441,446,723, \underline{12}, 841$

Wenhai Yan 16, 603

Wenzel E. $12,747,13,93,14,189$, $15,601,707,817$

Wessely P. 14, 339

White J.G. 11,339

Whittingstall P. $\underline{9}, 446,723, \underline{11}, 137, \underline{12}, 841$

Wichert v. P. 10,637

Wie Song 16, 603

Wienert V. 14, 547

Wieselcouch E.O. $\underline{9}, 973$

William-Ollson G. 12,229

Williams G. 10, 547, 14, 245

Wilmink J.M. 13, 465

Winkler H. 10, 81, 13, 55

Winlove C.P. 13,473

Witte S. $\underline{9}, 323,831, \underline{10}, 351,393, \underline{12}, 903$, $13,565,753$

Woimant F. 9,652

Wolf G. 12,79

Wolf H. 15,359

Wolf H.R.D. 10,393

Wolf S. $11,623,12,437,725, \underline{13}, 435, \underline{16}, 357$

Wong W.J. 14, 723

Woodburn K.R. 13, 515

Wu Ben-Jie 11, 459, 507

Wu He-Guang 11, 25

Wu Yunpeng 16, 135

Wurzinger L.J. 16, 623

Xiao Bao-guo 10, 309

Xieju X. 11, 237

Xu Jia-Ling 11, 459, 507

Xue Q.F. 16, 645

Xue Qu.ff. 15, 851

Yamaguchi S. $\underline{9}, 297$

Yamamoto J. 14, 509

Yamamoto K. 12, 297

Yamarat P. 15, 729

Yamazaki K. $\underline{9}, 376,11,251$

Yamazaki M. 13, 623

Yan Lu-Nan 11, 25
Yano S. $\underline{9}, 376,11,251$

Yarnell J.W.G. 12, 545

Ybema S.J. $\underline{9}, 1017$

Yedgar S. 14,497

Yin X.-J. 12, 831

Yoa R.G. 14, 663

Yokose T. 10, 463, 11, 175, 12, 935

Yokoyama M. 15, 89, 16, 143, 497

Yörükan S. 11, 121

Yoshikoshi A. 15, 889

Youji Suzuki $\underline{9}, 909$

Youtian H. 11, 237

Youtian Huang 16, 603

Yova D. 14, 369, 15, 107

Yu Gui-Fen 11, 459, 507

Yu J.J.H. 15, 775

Yuan G.-G. 11, 635

Yubero L. 14, S7

Yue D. 16,267

Zaninelli R. $\underline{9}, 55$

Zannad F. 121, 357

Zannad F. 9 , 219

Zeidler A. 9, 935

Zeiler K. 10, 499, 14, 339

Zhang Ch. 16, 267

Zhang X.-J. 12, 461

Zhang Y.-L. 12, 461

Zhang Yan 9,615

Zhao Ching 9, 374, 615

Zhao R.-R. 14, 779

Zhao Yu-Heng 11, 459, 507

Zhong Gui-Hui 11, 25, 635

Zhou D. 11, 635

Zhou R. 12, 593

Zhu J.-C. 9, 897, 11, 113

Zhuang F.-y. 15, 851

Zhuang F.Y. 16, 645

Zickmann B. 12, 71

Ziegler O. 12, 873

Zijlstra W.G. $\underline{9}, 553$

Zilow E.P. 14, 233

Zimmermann R. $\underline{9}, 125$

Zkhiri F. 13, 481, 15, 325

Zoellner K. $9,460,10,653$

Zong W. 12, 593

Zoukourian C. 14, 63, 16, 661

Zurborn K.H. $\underline{9}, 328$

Zwaginga J.J. 14, 769

Zyma S.A. 10, 231 


$\begin{array}{llllllllllll}\mathrm{S} & \mathrm{U} & \mathrm{J} & \mathrm{E} & \mathrm{C} & \mathrm{T} & \mathrm{I} & \mathrm{N} & \mathrm{D} & \mathrm{E} & \mathrm{X}\end{array}$

Abstracts

10th Annual Meeting of Deutsche Gesellschaft für klinische Mikrozirkulation und Hämorrheologie, Mainz, Germany 1991 12, 465

11th Annual Meeting of Deutsche Gesellschaft für Kiinische Mikrozirkulation und Hämorheologie, Heidelberg, Germany 1992 13, 807

13th Annual Meeting Japanese Society of Biorheology, Nara City, Japan 1990 10, 665

14th Annual Meeting Japanese Society of Biorheology, Tokyo, Japan 1991 11, 667

15th Annual Meeting Japanese Society of Biorheology, Gifu, Japan 1992 13, 97

16th Meeting of the Japanese Society of Biorheology, Tokyo, Japan 1993 13, 707

1st International and 8th European Conference on Clinical Hemorheology, Vienna, Austria 1993 $\underline{13}, 593,715$

2nd Scandinavian Symposium on Clinical Haemorheology, Uppsala, Sweden 1992 13, 269

6th Joint Meeting on Clinical Hemorheology French Society of Biorheology $\underline{9}, 159$

7ème Réunion de la Societé d'Hémorhéologie de l'Ouest, Rennes, France 1990 11, 513

8th International Congress of Biorheology, Yokohama, Japan 1992 12, 599

9th European Conference on Clinical Hemorheology, Siena, Italy $1995 \underline{15}, 399$

Annual Meeting of the German Society for Clinical Microcirculation and Hemorheology, Aachen, Germany 1994 15, 235
Beijing Satellite Symposium of the 8th International Congress of Biorheology, Beijing, China $1992 \underline{12}, 769$

Chinese National Congress on Clinical Hemorheology and Medical Biophyico-Chemistry, Shanghai, China 1994 14, 847

Congress of the French Society of Fundamental and Clinical Biorheology, San Malo, France 1993 14, 287

International Symposium on Biofluid Mechanics and Biorheology, Munich, Germany 1989

$\underline{10}, 119$

Microsymposium: Dynamic Rheologic

Measurements in the $\mathrm{c}+\mathrm{A} 831$ linic

$\underline{9}, 327,328,329$

Report on the Euromech Colloqium 311, Berlin, Germany 1993 14, 421

Sixth European Conference on Clinical Hemorheology, Frankfurt/Germany 1989, ㅇ, 1033

4th International Symposium on Clinical Haemorheology, Portugal $\underline{9}, 641$

a-tocopherol nicotinate $\underline{15}, 775$

acid probes $\underline{11}, 4 \underline{9}, 107$

acute infections $\underline{9}, 199$

acute phase proteins $\underline{15}, 763$

acute-phase response 12,797

adenosine $\underline{14}, 709,15,221$

adenosine diphosphate $\underline{13}, 623$

adhesiography $\underline{10}, 401$

adhesion $\underline{9}, 855, \underline{12}, 817$

adhesion molecules 14,379

ADP inducing platelet reaction 10, 309

adrenergic receptors 14 , S 37 
adsorption $\underline{9}, 695$

age $\underline{10}, 499$

aggregate size distribution $\underline{14}, 497$

aggregation $\underline{9}, 313, \underline{10}, 297$

aggregation index 10,363

aggregation kinetics 14,497

aggregometry $\underline{12}, 733$

air pollution 10,485

albumin $\underline{9}, 695,7 \underline{15}, \underline{11}, 167, \underline{12}, 761$

alpha2-macroglobulin 14, 127

anaemia $\underline{9}, 1023, \underline{11}, 605, \underline{12}, 499,587,883$,

$13,491,519$

analgesics 14,339

analytical variation $\underline{10}, 525$

angina $\underline{9}, 219$

angina pectoris $\underline{9}, 923$

angiopathy $\underline{11}, 243$

angiotensin-converting enzyme inhibitor $\underline{15}, 335$

animal model $12,689,11,465$

anterior ischemic optic neuropathy 11,623

anticoagulant $12,831,935$

antiplatelet $\underline{12,831}$

antithrombin III 12,947

antithrombotic agents $\underline{9}, 339$

aortic valve replacements $\underline{9}, 139$

apheresis 12,705

apolipoprotein A-I and B $\underline{15}, 763$

apolipoproteins $\underline{12}, 549$

arterial thrombosis $\underline{9}, 339$

ascorbic acid 14, S7, 15, 107

asphyxia 14,233

aspirin 15,877

atherogenesis $\underline{12}, 805$

atherosclerosis $\underline{12}, 817, \underline{13}, 671$

$14,+$ A86 181, 203

automation 9,593

autotransfusion $\underline{10}, 547$

Baxtroxobin 15, 89

Behcet's disease 15, 157

bezafibrate $\underline{13}, 679$

biliary cirrhosis $\underline{13}, 491$

blood cell electrophoresis 10,309

blood cell filtration 11,317

blood density $\underline{9}, 319$

blood doping 12, 505

blood elasticity 14, 339

blood filtration 10,547

blood flow structuring in microvessels $\underline{15}, 783$

blood flow velocity $\underline{13}, 435$

blood fluidity $14,189,355$

blood groups $\underline{12}, 579$

blood lactate $\underline{13}, 187 \underline{15}, 147,201$

blood non-Newtonian behaviour 14,789 blood rheological disorders $\underline{15}, 697$

blood rheology $9,897,923,935,10,65,275,321$

$637,11,63,79,167,175,623,641,12,341,401$, $407,437,13,237,719,743$,

blood sedimentation $\underline{9}, 35$

blood stasis 14,463

blood substitute 12,733

blood viscoelasticity $\underline{9}, 307,569, \underline{10}, 363,463$,

11,175

blood viscosity $\underline{9}, 35,41,81,101,1 \underline{15}, 14 \underline{9}, 181$,

$19 \underline{9}, 583,593,603,633,781,953,983,10,65$

$171,185,215,309,463,601,11, \underline{9}, 25,107$,

$175,317,441,447,12,381,49 \underline{9}, 14,105,33 \underline{9}$,

$463,473,51 \underline{9}, 637,677,685,697,723,769$,

$15,25,133,201,213,729$

blood viscosity autoregulation factor 15,61

blood volume 10,375

breast cancer $\underline{15}, 311$

buffer $\underline{11}, 317$

buflomedil 15,221

Ca-channels 14,545

calcium $\underline{9}, 273,723, \underline{10}, 81$

calcium antagonist $\underline{15}, 707$

calcium-dobesilate $\underline{11}, 1$

cancer 15,755

capillary flow $\underline{15}, 877$

capillary resistometry $14, \$ 47$

carbon monoxide 12,593

cardiac assist devices 11,325

cardiac disorders $\underline{9}, 797$

cardiac function test $\underline{10}, 205$

cardiac index $\underline{14}, 329, \underline{15}, 867$

cardiac load 15,347

cardiac output 13,719

cardiac rehabilitation $\underline{10}, 423$

cardiovascular disease $\underline{10}, 435, \underline{14}, 171$

carotid atherosclerosis $\underline{14}, 841$

catecholamines $\underline{9}, 55, \underline{14}, 369$

cell age $\underline{9}, 723$

cell changes $\underline{14}, 19$

cell culture 11,417

cell membrane $\underline{12}, 649$

cell orientation $\underline{9}, 791$

cell protection $14, \mathrm{~S} 15$

cell separation $\underline{13}, 55$

cell transit analyser 15, 107

cell viscosity $\underline{11}, 19$

cell volume $\underline{10}, 653, \underline{11}, 295, \underline{12}, 677$

central artery occlusion hemodilution $\underline{12}, 883$

cerebral blood flow $\underline{11}, 9, \underline{13}, 245, \underline{14}, 27$

cerebral chronic ischemia 12,697

cerebral infarction $\underline{10}, 5 \underline{15}, \underline{13}, 253, \underline{15}, 89,783$

cerebral ischemia $\underline{13}, 623$

cerebrovascular stroke $\underline{12}, 883$ 
channelyzer $\underline{15}, 889$

chemotactic stimulation 15,157

Chinese traditional medicinal herbs 10,309 chinoin $\underline{9}, 865$

cholesterol $\underline{9}, 149, \underline{10}, 95, \underline{12}, 54 \underline{9}, 705$

cholesterol hemisuccinate 11,583

chronic cor pulmonale 15,851

chronic obstructive arteriosclerotic disease 10, 225

chronic renal failure $\underline{11}, 4 \underline{9}, \underline{14}, 213$

chronic venous insufficiency 14, S53, S65

claudication 10,321

coagulation $\underline{12}, 49 \underline{9}, 14,463$

compaction stasis $\underline{9}, 751$

computerized image analysis 14,497

congestive heart failure $\underline{15}, 335, \underline{15}, 347$

conjunctiva $\underline{13}, 435$

contraceptives 13,435

contrast media $11,91, \underline{12}, 357,381,393,401$

cordocentesis $13,215,14,637$

coronarography $\underline{9}, 885$

coronary artery disease 15,737

coronary circulation 11, 121, 14, 779

coronary heart disease $10,423, \underline{14}, 127$

coronary stenosis $\underline{9}, 885$

coronary syndromes $\underline{10}, 601$

corticosteroids $\underline{13}, 237$

Couette viscometer 15,45

Coulter counter $\underline{15}, 889$

cutaneous microangiopathy 15,817

Cyclo 3 Fort 14, S53

cyclosporin-A $13,49,465$

cytochalasin B $\underline{9}, 69$

cytosolic red cell $\mathrm{Ca} 2+$ content 11,617

cytotoxic drugs $\underline{15}, 311$

Defibrinogenation 15,89

deformability/passage analyzer $\underline{10}, 653$

degradation products $\underline{13}, 13$

denbufylline 10,215

density $\underline{10}, 375$

depletion 9,695

dermatomyositis 15,627

dexamethasone 15,763

dextran $\underline{9}, 1,723, \underline{10}, 383, \underline{11}, 339, \underline{13}, 779$,

14,531

dextrans $\underline{10}, 287$

diabetes mellitus $\underline{9}, 101,281,351,361,559,603$,

$615,791,845,855,935,965,989,1017,10,557$

$561,11,155,167,183,191,205,227,237,243$,

$405,649,12,437,449,713,787,935,13,147$

$253,661,14,63,83,473,481,489,539$,

$15,3,25,53,73,311,657$

diabetes type $1 \underline{12}, 873, \underline{14}, 473,481$

diabetes type $2 \underline{10}, 477$ diabetic angiopathy $\underline{10}, 463, \underline{11}, 597$

diabetic arteriopathy 14,413

diabetic microangiopathy $\underline{11}, 1,175,217, \underline{12}, 437$

diabetic plasma 11,387

diabetic therapy $\underline{11}, 243$

diet $\underline{9}, 1 \underline{15}, \underline{11}, 107$

diffractometry $\underline{13}, 641, \underline{15}, 177$

dimethyl xanthines $\underline{14}, 545$

dipyridamole $\underline{10}, 263$

disseminated sclerosis $\underline{11}, 281$

dopamine $\underline{9}, 1029$

Doppler-Ultrasound $\underline{13}, 729$

dose-effect relation $\underline{15}, 649$

drug screening 13,3

drug therapy 10,473

drug treatment $\underline{9}, 831$

drug-withdrawal therapy $\underline{14}, 339$

Ektacytometer 11, 459, 14, 819

ektacytometric osmoscan curve 11, 507

ektacytometry 14, 279, 605, 619

elastase $\underline{9}, 149$

electrophoretic mobility $11,281,13,473$

elimination kinetics 14,189

endothelin 14, S37

endothelium $\underline{9}, 855, \underline{10}, 241, \underline{12}, 817$

14, S37, 19,63

endotoxin $\underline{15}, 749$

endurance training 10,423

energy metabolism $\underline{13}, 55$

enzyme activities 11,35

epidemiological study $\underline{12}, 549$

epidemiology $12,545,13,83$

ergometry 10,393

ergopeptide alkaloids 15,133

eryhtrocyte sub-populations $\underline{9}, 897$

erythrocyte $\underline{9}, 1,125,695,855,973,12,587$,

14,149

erythrocyte adherence $\underline{10}, 241$

erythrocyte aggregation $9,41,715,739,781,909$,

$10,473,515,601,11,175,205,351,641,649$,

$12,381,393,761,873,13,49,215,253,261$,

$775,779,14,11,509,605,637,643,15,13,97$,

$147,191,201,273,291,311,359,381,697,783$,

$829,16,105,143,313,549,685$

erythrocyte aggregation in newborns $\underline{9}, 823$

erythrocyte centrifugal packing 13,519

erythrocyte cytosolic $\mathrm{Ca} 2+15,613$

erythrocyte deformability $\underline{9}, 181,247,351,361$,

$559,577,615,625,885,897,1009,1017,1023$

$10,35,43,255,263,275,485,535,561,11,19$,

$79,113,121,133,205,325,351,497,583$,

$12,381,573,787,841,13,3,49,67,73,187$,

$201,215,261,459,465,481,491,641,651,661$, $743,767, \underline{14}, 347,605,619,637,779,831$, 
$15,13,147,201,213,273,677,749,797,829$ $851,859,877,16,105,117,135,271,515,533$ erythrocyte disaggregation $\underline{10}, 473$ erythrocyte filterability $9,89,209,965,10,505$, $11,479,13,237,661,767,791$ erythrocyte filterability in newborns $\underline{10}, 113$ erythrocyte filtration 14,347 erythrocyte flexibility 11,191 erythrocyte fluidity $\underline{15}, 133$ erythrocyte fragibility $\underline{9}, 273$ erythrocyte indices $\underline{15}, 797$ erythrocyte membrane $\underline{9}, 999, \underline{10}, 485, \underline{13}, 237$, $14,819,831$

erythrocyte membrane fluidity $11,617, \underline{13}, 227$, $15,613,851$

erythrocyte membrane lipids 13,35

erythrocyte membrane phospholipids 10,449

erythrocyte morphology 10,231

erythrocyte properties 9,723

erythrocyte rheology $\underline{14}, 213,497$

erythrocyte rigidity 13,501

erythrocyte sedimentation-rate $\underline{9}, 319, \underline{11}, 501$, 14,509

erythrocyte sialic acie 10,477

erythrocyte surface charge 13,775

erythrocyte viscoelastic properties $\underline{15}, 1^{\prime \prime} 7$

erythrocyte viscosity 11,205

erythrocyte volume distribution $\underline{15}, 889$

erythrocytes $\underline{10}, 103, \underline{11}, 295,383, \underline{13}, 67,407$, $473,14,63$

erythropoietin $\underline{10}, 653, \underline{12}, 505, \underline{14}, 53$

esoc conservation medium 10,43

ESR equation coefficient $\underline{9}, 41, \underline{10}, 309$

ethinyl estradiol 13,435

ethnicity $\underline{13}, 737, \underline{14}, 697$

exercise tolerance test $\underline{10}, 423$

exertion $\underline{12}, 511$

Familiar hypercholesterolemia 15,677

Felodipine 14, 355

female NIDDM 15,775

fenofibrate $\underline{10}, 297$

fetal blood 13, 215, 14, 93, 637

fibrin degradation products $\underline{13}, 515$

fibrin elasticity 10,401

fibrinogen $\underline{9}, 553,695,845,909,935,1017$,

$10,297,309,339,11,191,387,623,12,455$,

$535,545,639,705,761,787,805,947, \underline{13}, 13$,

$147,245,679,737,14,37,93,171,181,245$,

$401,481,531,15,3,97,311,381,737,841$

fibrinolysis $\underline{10}, 541$

fibrinolytic activity $\underline{15}, 897$

filterability $\underline{10}, 353,477, \underline{11}, 295, \underline{14}, 149,489$

filterability index $\underline{12}, 427$

filtration $\underline{9}, 973$ filtration test $\underline{12}, 747$

filtrometry 12,733

fish red blood cells 12,677

flexibility index 10,363

flow instability $\underline{\underline{9}}, 989$

fluid shifts 11,429

fluorescence $\underline{9}, 999$

fluorescence spectroscopy 11,49

fluorescent parameters $\underline{11}, 1$

fluorocarbon emulsion $\underline{12}, 733$

foot ulcer $\underline{15}, 73$

Gangliosides 14,347

gelatins $\underline{10}, 287$

gemfibrozil 10,329

gender 10,499

Ginkgo biloba extract 9 , 323, 15, 649

glucose 11,35

glutathione $\underline{9}, 209$

glycated erythrocyte membrane proteins $\underline{11}, 405$ glycation 14,63

glycemic control 12,437

glycerol $10,185,515$

glycoalyx 14, 531

glycoprotein $\underline{9}, 331$

glycosylated fibrinogen 11,649

granulocyte filterability 13,501

gravitational sedimentation $\underline{9}, 751$

H.E.L.P. $13,701,14,27,37,45,181$

haemoglobin 11, 361

halothane anesthesia 10,65

haptoglobin $\underline{13}, 147, \underline{14}, 481$

HDL subfractions 15,667

head and neck cancer 15,37

hearing loss 10,51

heart function 14,779

heart infarction 12,545

heart pumping efficiency 10,411

heat generation 10,411

Heinz bodies 14, 557

helium-neon laser 14,369

hematocrit $\underline{9}, 361,55 \underline{9}, 583,823,953,989,1023$

$11,121,623,635,12,437,455,535,883$,

$\underline{13}, 187,14,105,245,305,321,557,789$,

$15,37,147,201,841$

hematology $\underline{10}, 525, \underline{11}, 303, \underline{12}, 627$

hemodialysis $10,637,13,67$

hemodilution $10,205,383, \underline{13}, 719,779, \underline{14}, 11$,

$189,15,601$

hemoglobin $10,81,14,585$

hemoglobin concentration 14,677

hemoglobin sedimentation 12,587 
hemoglobinopathy $\underline{15}, 177$

hemolytic anemia $\underline{14}, 557,571$

hemorhagic stroke 10,309

hemorheolgoy 14,271

hemorheological parameters $\underline{9}, 625, \underline{11}, 405,657$

hemorheological pattern 12,573

hemorheology $\underline{9}, 27,101,109,219,603,739$,

$845,953,983,989,10,231,329,423,463,515$,

$525,541,625,11,25,107,227,243,303,339$,

$383,397,429,441,465,597,12,415,499,511$.

$521,593,657,689,733,13,83,177,187,201$,

$215,253,447,651,679,687,14$, S53, 11, 45, 75, 93,305

hemorheometer 13,767

hemoviscometry $\underline{12}, 415$

heparin $\underline{10}, 263, \underline{12}, 747, \underline{15}, 633$

hereditary spherocytosis $\underline{14}, 571, \underline{15}, 177$

heredity 11,243

hesperidin methyl chalcone $\underline{14}, \mathrm{~S} 7$

hirudin $\underline{12}, 747$

human hepatoma cells $\underline{15}, 763$

hydric intake 12,511

hydroxethyl starch $\underline{10}, 287,383, \underline{13}, 729,779$,

$14,11,189$

$\underline{\underline{4}}$ hypercholesterolemia 15,841

hypercholesterolemic rabbits $\underline{9}, 149$

hyperglycemia $\underline{9}, 935, \underline{11}, 167, \underline{14}, 271$

hyperlipoproteinemia $\underline{10}, 297, \underline{13}, 447, \underline{15}, 867$

hyperosmolar agent $\underline{10}, 515$

hyperoxic environment 15,797

hypertension $\underline{9}, 8 \underline{9}, 101, \underline{10}, 95,477,491$,

$\underline{12}, 427,579, \underline{13}, 35,14,355, \underline{15}, 657,697,707$

hyperthyroidism $\underline{9}, 577$

hypertriglyceridemia 15,841

hyperviscosity $\underline{10}, 51$

hypoglycaemia $\underline{9}, 603$

hypothyroidism $\underline{10}, 255$

hypoxanthine oxidase $\underline{15}, 641$

hypoxia $14, \mathrm{~S} 1, \mathrm{~S} 15$

hypoxic and ischemic microcirculation $\underline{15}, 133$

Idebenone 11, 351

iloprost $\underline{10}, 171,15,829$

immunoglobin 15,359

immunoglobulins $\underline{9}, 715, \underline{14}, 697$

immunosuppressive drugs $\underline{14}, 663$

impedance plethysmography $\underline{15}, 311$

impotence 11, 227

in vitro incubation $\underline{15}, 613$

in vivo-ridification of erythrocytes $\underline{10}, 625$

inflammation 11, 465

instrument evaluation $\underline{10}, 275$

insulin $\underline{9}, 603, \underline{12}, 787$

interleukin-6 15, 763

intermittent claudication $\underline{9}, 1017, \underline{10}, 225$ interstitial fluid $\underline{12}, 903$

intracellular electrolytes $\underline{15}, 797$

intracellular sodium concentration $\underline{9}, 89$

intraerythrocytic calcium $\underline{12}, 527, \underline{14}, 797$

intraoperative autotransfusion $\underline{10}, 547$

intravenous anaesthesia $\underline{10}, 65$

iodine 11,91

iohexol 11,91

ionic and non-ionic contrast media 12,341

iron deficiency 11,605

ischaemia $\underline{11}, 133,479, \underline{12}, 527, \underline{13}, 155,687$,

$15,221,627$

ischaemic heart disease $\underline{9}, 885, \underline{10}, 241, \underline{11}, 397$,

$14,329, \underline{15}, 867$

ischaemic stroke $\underline{10}, 309, \underline{11}, 635, \underline{14}, 723$

Jaundice $\underline{15}, 273$

Laboratory parameters $\underline{9}, 307$

lactoferrin 14, 379

laser-doppler electrophoresis $\underline{10}, 103$

LDL 15,667

LDL-apharesis $\underline{9}, 81, \underline{11}, 9$

leech treatment $\underline{9}, 569$

leukocyte $14,63,149$

leukocyte adhesion molecule $\underline{12}, 713$

leukocyte adhesivity 12,747

leukocyte deformability $\underline{11}, 79, \underline{14}, 233$

leukocyte filtration $\underline{11}, 369,14,597, \underline{15}, 53$

leukocyte rheology 11,369

leukocytes $\underline{10}, 157, \underline{11}, 129,383, \underline{12}, 535,857$,

$\underline{13}, 73,155,407, \underline{14}, 539,591$

leukotriene $\underline{9}, 209$

light back-scattering $\underline{9}, 739$

light transmission 12,841

ligustrazine $\underline{9}, 615, \underline{10}, 205$

lipid $A \underline{13}, 45$

lipid peroxidation $\underline{11}, 497, \underline{14}, 591$

lipid peroxides 15,677

lipids $\underline{9}, 257, \underline{14}, 203$

lipophilicity $\underline{15}, 877$

lipoproteins $\underline{15}, 737$

low flow rate $\underline{9}, 297$

loxaglate 12,357

lymph 12, 903

lymphphocyte fluidity $\underline{9}, 47$

Macroglobulinemia $\underline{12}, 725$

macromolecular permeability $14, \mathrm{~s} 23$

magnetic resonance imaging $\underline{11}, 281$

malaria 15,729

malonyldialdehyde $\underline{15}, 641$ 
mammals $\underline{12}, 63 \underline{9}, \underline{15}, 291$

Mathew scale 14, 37

maturity onset diabetes 14,271

mechanical fragility $\underline{14}, 557$

mechanical properties 10, 561

medical leech 15,633

membrane elasticity $\underline{9}, 313$

membrane fluidity $\underline{9}, 839,11,583$

membrane lipids $\underline{14}, 131, \underline{15}, 311$

membrane viscosity $\underline{9}, 313$

menopause 11, 397

menstrual cycle $\underline{10}, 21$

mesoglycan 12,449

metabolic control 11,405

methodological comparison 11,575

methylxanthines $\underline{9}, 69$

microalbuminuria $\underline{9}, 351$

microangiopathy $\underline{11}, 237,405,14,271, \underline{15}, 3$

microcirculation $\underline{9}, 797,10,393,601,625$,

$11,129,217,237,479,597,12,725,903$,

$\underline{13}, 435,14,519,677,57,15,707$

microcirculation hemodynamics 11,417

microcirculation of the skin $\underline{15}, 649$

microcirculatory stases in brain 15,783

microcomputer 10,263

microhematocrit 12,341

micropipette $\underline{11}, 383, \underline{13}, 407, \underline{14}, 489$

micropipette aspiration $\underline{13}, 671$

microrheological determinants 10,449

microrheology $\underline{9}, 999$

microscopic photometric monolayer technique 15,133

microsieve $\underline{9}, 125$

microspherocyte 14,571

microvascular disease 14,539

microvessel diameter $14, \mathrm{~S} 23$

molar substitution 14,11

molecular collagen $\underline{10}, 411$

monoclonal dysglobulinemia $\underline{9}, 877$

monocyte $\underline{12}, 817, \underline{14}, 149$

morphology of RBCs 15,851

mountain sickness 11,303

MRI white matter disease $\underline{14}, 841$

multiple linear regression $\underline{9}, 307$

multiple sclerosis $11,281, \underline{12}, 947$

multivariate analysis 11,387

muscle perfusion 10,393

myelodysplastic syndromes $\underline{13}, 767$

myocardial infarction $\underline{9}, 257,923, \underline{11}, 63$,

$\underline{15}, 367,715$

myocardial oxygenation 12,883

myocardial perfusion $\underline{10}, 411$

myocardial ultrastructure $\underline{14}, 779$

myocardium $\underline{15}, 97$
$\mathrm{Na} / \mathrm{K}$ cotransport system $\underline{9}, 89$

naftidrofuryl $\underline{9}, 339$

neonatal blood $\underline{14}, 585$

neonatal polycythemia 14,321

neonates $\underline{14}, 659$

Nephrotic syndrome 14, 769

nephrotic syndrome in Nigerians $\underline{15}, 897$

neuraminidase $\underline{13}, 775, \underline{14}, 279,531$

neurosurgery 13,459

neutrophil $\underline{9}, \underline{6} \underline{9}, 973, \underline{14}, 233, \underline{15}, 157,221$

neutrophil filterability $\underline{13}, 23$

NIDDM $\underline{15}, 859$

nimodipine 12,697

nitrate $\underline{13}, 481, \underline{15}, 335$

nitrite $\underline{13}, 481$

non-insulin-dependent diabetes mellitus (NIDDM)

15,859

non-Newtonian behaviour 15,755

non-Newtonian behaviour index ' $\mathrm{N}$ ' 14,685

norepinephrine $\underline{9}, 1029$

nutrition $\underline{15}, 213$

O-(B-Hydroxethyl)-rutosides 10, 231

obesity $\underline{9}, 983, \underline{10}, 541, \underline{11}, 641, \underline{12}, 573, \underline{14}, 203$

occlusive angiopathy $\underline{9}, 965$

okadaic acid 14,819

omega- 3 fatty acids $\underline{10}, 157, \underline{15}, 867$

omega-3 polyunsatured fatty acids $\underline{14}, 663$

oncotic agent $\underline{12}, 733$

oral contraceptives $10,21,499$

orbitometer 10,401

organ blood flow 10, 383

organic phosphates 14,53

oscillary capillary viscometer 11,575

oscillating capillary rheometer $\underline{9}, 307, \underline{10}, 363$,

14, 509

osmolality 11,91

osmostic pressure 11,507

osmotic fragility $\underline{9}, 257, \underline{12}, 947, \underline{14}, 557$

osmotic pressure 15,889

oxidant attack 14,591

oxygen delivery $\underline{9}, 149$

oxygen free radical $\underline{11}, 361, \underline{15}, 641$

oxygen transport 10, 383

oxygen uptake 10,393

Pancreatitis 11, 25

paraproteinemia 14, 401

passage time distribution 11,369

pentoxifylline $\underline{9}, 47,69,351,973, \underline{10}, 215,225$,

$11,227, \underline{12}, 713,14,379,413$

percutaneous transluminal coronary angioplasty $\underline{13}, 679, \underline{15}, 737$ 
perihemorheology $\underline{12}, 903$

perinatal asphyxia 14,75

peripheral arterial disease $10,339, \underline{12}, 867,883$,

$13,515,14,181,413,15,191,681$

peripheral hemodynamics 14,709

peripheral vascular disease $\underline{9}, 47,11,479$,

$\underline{12}, 521,13,73$

permeability 12,903

pharmacology $\underline{9}, 855$

phase separation $\underline{9}, 751$

phocid seals $\underline{12}, 657$

phosphate buffered saline 11,317

phospholipids 10, 95

phosphorylation 14, 819

physiological variation $\underline{10}, 525$

piyavit 15,633

plasma albumin $\underline{14}, 481$

plasma density $\underline{9}, 319$

plasma exchanges $\underline{9}, 877$

plasma fibrinogen $\underline{12}, 427, \underline{14}, 841, \underline{15}, 697$

plasma lipids $\underline{12}, \underline{557}$

plasma protein composition 14,769

plasma proteins $\underline{9}, 199, \underline{12}, \underline{557}, 873, \underline{14}, 697$

plasma rheology $\underline{10}, 637$

plasma substitutes 10,287

plasma thrombomodulin $14, \mathrm{~S} 1$

plasma trapping 15,729

plasma viscosity $9,35,101,237,319,543,553$,

$569,633,831,861,877,1017,10,21,51,65$,

$165,185,309,339,383,435,11,167,217,351$

$447,501,623, \underline{12}, 437,545,54 \underline{9}, 557,639,725$,

$\underline{13}, 261,77 \underline{9}, \underline{14}, 11,37,401,697,769, \underline{15}, 3,45$,

$305,311,359,737,755$

plasma volume $\underline{10}, 51$

plasmapheresis $\underline{14}, 181$

plastic deformation 14,659

platelet aggregation $\underline{9}, 109,139,219,615$,

$10,225,11,351, \underline{12}, 947, \underline{13}, 155,177,623$, $\overline{14}, 223,709,15,633$

platelet cytosolic calcium 14, 393, 15, 657

platelet filterability $\underline{12}, 935$

platelet function 11,339

platelet membrane fluidity $\underline{14}, 393, \underline{15}, 657$

platelet membrane lipids 14,393

platelet retention $\underline{12}, 947$

platelet shape change 10,401

platelet volume 12,935

platelets $\underline{9}, 331, \underline{12}, 499,857, \underline{14}, 463$

polbax $\underline{15}, 641$

polycythemia $\underline{9}, 1023, \underline{14}, 585$

polymorphonuclear cytosolic calcium 14,597 , $\underline{15}, 53$

polymorphonuclear endothelium adhesion 11,417

polymorphonuclear leukocytes $14,379,489$

polymorphonuclear membrane cholesterol /

phospholipid ratio $\underline{15}, 53$ polymorphonuclear membrane fluidity $\underline{14}, 597$, $\underline{15}, 53$

pore size 11, 479

post renal transplant erythrocytosis $\underline{13}, 261$

post-thawing conservation 10, 43

postaglandins 15,829

pre-eclampsia $\underline{13}, 719$

pre-eclampsic toxaemia 10,491

pregnancy $\underline{10}, 491, \underline{12}, 427, \underline{13}, 147,501$

probit analysis 14,223

prolactin 11, 227

protein concentration $\underline{10}, 375$

proteins $\underline{9}, 257$

psoriasis $\underline{13}, 49$

pyruvate kinase deficiency $\underline{13}, 55$

Rat blood $\underline{10}, 215$

rat model 10,231

red blood cell aggregation $\underline{9}, 1,237,281,543$,

$723,771,797,923,935,10,411,423,557$,

$11,623,635,12,341,437,455,535,657$.

$14,53,83,117,237,519, \mathrm{~S} 53,15,3,305,667$

red blood cell calcium content $\underline{13}, 35,227$

red blood cell deformability $\underline{9}, 27,55,14 \underline{9}, 297$,

$1029,10,95,11,55,217,295,317,12,649,677$, $757,841,13,45,55,671,14,53,321,591,769$, $15,107,619,641,775$

red blood cell disaggregation $\underline{9}, 845$

red blood cell elasticity $\underline{13}, 671$

red blood cell filterability $\underline{9}, 237,543,861,10,21$,

$463,11,91,12,357,14,245,619,685$

red blood cell flux $\underline{10}, 505$

red blood cell fragility $\underline{14}, 659$

red blood cell internal viscosity $\underline{15}, 729$

red blood cell lipids 13,447

red blood cell membrane $\underline{10}, 653$

red blood cell membrane tanktreading $\underline{9}, 751$

red blood cell mobility tests $\underline{11}, 281$

red blood cell morphology $12,341,357,14,797$

red blood cell orientation $\underline{15}, 619$

red blood cell rigidity 12,461

red blood cell sedimentation 14,519

red blood cell shear flow $\underline{9}, 1023$

red blood cell suspension $\underline{9}, 313$

red blood cell transport 14,789

red blood cell volume 12,757

red blood cells $\underline{10}, 157,353, \underline{11}, 339, \underline{12}, 677$,

$14,131,369,557,571$

regression analysis $\underline{9}, 583,633$

relative resistance $\underline{9}, 297$

relative viscosity $\underline{14}, 769$

renal failure $\underline{13}, 67$

renal transplant $13,465,14,663$

restenosis $\underline{13}, 679, \underline{15}, 737$

reticulocytosis $\underline{11}, 19$ 
retinal blood flow 12,725

retinal microcirculation $\underline{15}, 775$

retinopathy $\underline{9}, 281$

retinopathy of prematurity 15,305

rheological determinants $\underline{13}, 35$

rheological occlusion $\underline{9}, 751$

rheology $\underline{9}, 181,237,247,273,351,361,861$,

$1009,10,35,297,535,11,113,309,317,663$

$12,797,805,13,3,407,14,539,545$

rigidity of red blood cell 15,729

rotational viscometer 11,575

rouleaux $\underline{9}, 695, \underline{11}, 191, \underline{12}, 761, \underline{13}, 473, \underline{14}, 531$

rouleaux formation 14,697

Ruscus extract $14, \mathrm{S7}, \mathrm{S15}, \mathrm{S} 23$

S-adenosylmethionine $\underline{13}, 491$

salicylates $\underline{15}, 877$

selectin 12,817

sensorineural 10,59

sequential analysis $\underline{15}, 273,291$

serotonin antagonists 10,321

serum proteins $\underline{10}, 51$

shear activation $\underline{9}, 331$

shear elastic modulus $\underline{10}, 653$

shear forces 12,841

shear rate 15,667

shear stress 13,623

shear thinning $\underline{10}, 505$

sickle cell $\underline{12}, 415,587, \underline{13}, 519, \underline{14}, 545$

sickle cell anaemia $\underline{9}, 247,1009, \underline{10}, 35,535$,

$\underline{12}, 797, \underline{13}, 3$

Single-Photon-Emission computed tomography

$\underline{12}, 697$

sodium erythrocyte content 15,325

solubility $\underline{10}, 81$

sound speed 10,375

splenic conditioning 14,571

sports anemia 11,429

sports physiology 11,429

standardization 9,593

stomatocyte $\underline{15}, 133$

strain of flow 10,401

streptokinase $\underline{11}, 63, \underline{15}, 367$

stroke $\underline{9}, 273, \underline{10}, 185, \underline{11}, 167, \underline{15}, 715$

sulfur dioxide $\underline{10}, 485, \underline{11}, 497$

superoxide anions 14, 379

surface pO2 blood viscosity $\underline{10}, 393$

suspending medium 11, 459

suspending medium viscosity $\underline{15}, 619$

systemic lupus erythematosus 11.501

Tert-butyl hydroperoxide $\underline{15}, 107$

testosterone 11, 227
Thalassemia $\underline{15}, 177$

thalassemic syndromes $\underline{13}, 767$

theophylline 15,221

thixotropy $\underline{9}, 791, \underline{11}, 635, \underline{15}, 25$

thrombolysis $11,63, \underline{13}, 13$

thrombomodulin $\underline{15}, 627$

thrombus 12,593

ticlopidine $\underline{13}, 623, \underline{14}, 171$

tissue hematocrit 15,97

tissue oxygen pressure 14, 263

total red cell $\mathrm{Ca} 2+$ content 11,617

transcutaneous oxygen pressure 15191

transfusion $\underline{14}, 831$

transient ischemic attacks $\underline{9}, 109, \underline{11}, 35$

transient resistance $\underline{9}, 791, \underline{14}, 473$

transit time analysis $\underline{11}, 55$

trental 11,309

triglycerides $\underline{12}, 705$

trinitrine 13,481

troxerutine $\underline{9}, 543,871, \underline{11}, 597, \underline{14}, 83$

trypsin $\underline{13}, 473,775,14,531$

turpentine oil $\underline{11}, 465$

Ubbelohde viscometry $\underline{15}, 45$

ultrasound 14,117

ultrasound backscattering $\underline{9}, 771$

uremia 10,653

urokinase 11,63

Valvular disease $\underline{10}, 435$

varicose leg ulcers 14,379

vascular atherosclerotic disease $\underline{9}, 839, \underline{10}, 449$,

$11,617, \underline{12}, 449, \underline{13}, 701,14,393,597$,

$15,613,657$

vascular dementia $\underline{15}, 649$

vascular endothelium $14, \mathrm{~S} 1$

vascular resistance $\underline{13}, 215$

vasculitis $\underline{15}, 627$

vasoactive drugs 15,681

vein thrombosis $\underline{15}, 311$

venotonic drug $14, \mathrm{~S} 7$

venous insufficiency $\underline{9}, 871$

venous stasis $\underline{9}, 237,543,861$

vertebrate animals $\underline{12}, 649$

vertigo 15,601

video capillary microscopy $\underline{15}, 817$

video fluorescein angiography 12,725

viscoelastic phase angle $\underline{10}, 363$

viscoelasticity $\underline{9}, 313,935, \underline{10}, 499, \underline{12}, 593, \underline{15}, 25$

viscometer $\underline{14}, 401$

viscometry $\underline{11}, 575, \underline{12}, 733$

viscoreceptors $\underline{9}, 559$ 
viscosity $\underline{9}, 125,257,559, \underline{10}, 287,11,35,91$,

$237, \underline{12}, 705,761,831,947,14,53,75,93$,

15,715

von Willebrand Jürgens factor $13,515,623$

von Willebrand Jürgens syndrome $\underline{15}, 817$

Washed red cell filterability $\underline{9}, 27$

Weissenberg rheogoniometer $\underline{15}, 715$

Westergren method 14, 509

white blood cell adhesivity 14,83

white blood cells $10,353,11,309$

white blood cell plugging $\underline{9}, 297$

whole blood filterability $\underline{9}, 27, \underline{10}, 171,11,1,133$,

361,605

whole blood hyperviscosity 14,321

whole blood passage time $\underline{10}, 435$

whole blood viscoelasticity $\underline{9}, 323,831,15,649$

whole blood viscosity $\underline{9}, 615,10,21,435,11,217$,

$361,605,12,427,461,57 \underline{9}, 639,657,697$,

$13,13,187,215,201,215,237,473,491,515$,

$651,779,14,237,15,37$

wildlife health $\underline{12}, 627$

Xanthine oxidase $\underline{15}, 641$

Yield shear stress 13,701

yield stress $\underline{9}, 751, \underline{15}, 715$ 